\title{
FEEDBACK THEORY FOR TIME VARYING REGULAR LINEAR SYSTEMS WITH INPUT AND STATE DELAYS
}

\author{
SAID HADD, ABDELAZIZ RHANDI, AND ROLAND SCHNAUBELT
}

\begin{abstract}
We show that the class of regular time varying systems is invariant under perturbations by time-varying state and input delays. In particular, we give explicit formulas of the resulting input, output, and input-output maps. This result is used to solve the feedback problem for the delayed system. The relationship between the open and the closed loop system is investigated. Our results are applied to a parabolic boundary control problem with input and state delays.
\end{abstract}

S. Hadd, A. Rhandi, Department of Mathematics, University Cadi Ayyad, Faculty of Sciences, B.P.: 2390, 40000 Marrakesh, Morocco.

email: hadd@ucam.ac.ma, rhandi@ucam.ac.ma

R. Schnaubelt, Universität Halle, FB Mathematik und Informatik, 06099 Halle, Germany. email: schnaubelt@mathematik.uni-halle.de

2000 Mathematics Subject Classification. 34K30, 34K35, 47D06, 93C23, 93C25.

Key words and phrases. Regular time varying systems, retarded evolution equation, input delay, Lebesgue extension, feedback.

The support by DFG and CNRST is gratefully acknowledged. 


\section{INTRODUCTION}

We investigate retarded time varying linear systems of the type

$$
\begin{aligned}
w(t) & =A(t) w(t)+K(t) w_{t}+B(t) u(t)+L(t) u_{t}, \quad t \geq s \geq 0 \\
y(t) & =C(t) w(t), \quad t \geq s \geq 0 \\
w(s) & =x, \quad w_{s}=f, \quad u_{s}=g
\end{aligned}
$$

on Banach spaces $U, X$, and $Y$ (the control, state and observation space, respectively) with unbounded operators $A(t), B(t), C(t)$. We construct the corresponding input, output, and input-output maps and solve the feedback problem where the control is determined by $u(t)=\Delta(t) y(t)$, see Theorems 4.5 and 5.1. The delay terms in (nLDS) are given by

$$
K(t) w_{t}=\int_{-1}^{0} d k(t, \theta) w(t+\theta) \quad \text { and } \quad L(t) u_{t}=\int_{-1}^{0} d l(t, \theta) u(t+\theta)
$$

for operator-valued functions $\theta \mapsto k(t, \theta) \in \mathcal{L}(X)$ and $\theta \mapsto l(t, \theta) \in \mathcal{L}(U, X)$ of bounded variation. We assume that the system without delays (i.e., $K(t)=0$ and $L(t)=0$ ) is a regular well-posed time varying (or non-autonomous) system in the sense of Schnaubelt (2002b). This class contains systems arising from partial differential equations with point or boundary control and observation, see Schnaubelt (2002b) and Example 5.2.

Time invariant well-posed linear systems were defined by Salamon (1987) and Weiss (1989a, 1989b, 1994a). Weiss introduced the concept of 'regularity' in order to obtain good representation formulas for the input-output operator and the transfer function, see Weiss (1994a). Regular time invariant systems are stable under a large class of feedbacks due to Chapter 7 in Staffans (2004) and Weiss (1994b). The monograph Staffans (2004) gives a comprehensive account of the theory in the time invariant case, including non regular systems.

Much of the representation and feedback theory of regular systems has been extended to time varying systems in Schnaubelt (2002b). Throughout our paper we use the results from Schnaubelt (2002b), which are recalled in Section 2. There are only a few papers on time varying feedback theory for general linear systems with unbounded control and observation operators, cf. Schnaubelt (2002b). We want to mention in particular the works Hinrichsen and Pritchard (1994) and Jacob (1995) who solved time varying feedback problems working under different assumptions than in Schnaubelt (2002b). However, it seems that in their setting a satisfying control theory for the closed loop system is out of reach, see Schnaubelt (2002b) for a detailed comparison.

Typically the feedback mechanism does not act instantaneously so that it is reasonable to introduce delays in the system. For simplicity, we concentrate on the input delay term $L(t) u_{t}$ and the state delay $K(t) w_{t}$. The theory of time invariant control problems with delays has been developed at least since the 1960s. In the case of a finite dimensional state space $X$ we refer to e.g. Bensoussan et.al. (1992) and Vinter and Kwong (1981) for a detailed presentation and further literature. The infinite dimensional case, i.e., partial differential equations with delays in the state, input, or output, was investigated for instance 
in Da Prato and Lunardi (1990), Hale and Verduyn Lunel (2001), Jeong (1991), Nakagiri and Yamamoto (2001). These papers deal with the feedback stabilization problem of time invariant retarded infinite dimensional systems. In several recent contributions Kowalewski studied the optimal control of various classes of time varying retarded partial differential equations, see e.g. Kowalewski (2003), Kowalewski and Krakowiak (2001). The optimal control of time varying parabolic problems was treated in Acquistapace and Terreni (1999).

If one wants to investigate retarded problems within the usual framework of systems theory, one has to enlarge the state space in order to incorporate the prehistory of the state and of the input given by $w_{t}(\theta)=w(t+\theta)$, resp. $u_{t}(\theta)=u(t+\theta)$, for $\theta \in[-1,0]$ and $t \geq s$. In problems without control and observation one typically chooses the state space $C([-1,0], X)$. However, this choice is not convenient in control theory; for instance, one needs Hilbert spaces for optimal control problems. We use the so called 'extended state' $\left(x(t), x_{t}, u_{t}\right)$ introduced by Ichikawa (1982). This and alternative settings are discussed in Section 4.2.2 in Volume I of Bensoussan et.al. (1992). As a result we take the state space $\mathcal{X}=X \times L^{2}([-1,0], X) \times L^{2}([-1,0], U)$. All our results remain valid with the same proofs if one replaces here the exponent 2 by $p \in[2, \infty)$.

However, a point delay $\phi \mapsto \phi(-1)$ is not closable as a map from $L^{2}([-1,0], X)$ to $X$ so that it leads to an unbounded control or state operator. One can study finite dimensional, time invariant, retarded control problems in the framework of the Pritchard-Salamon class, a subclass of regular systems, see Pritchard and Salamon (1985). Retarded evolution equations without input or output were systematically investigated in the book Bátkai and Piazzera (2005) on an $L^{p}$ state space by means of the Miyadera perturbation theorem. As observed in Hadd and Idrissi (2005) and Hadd et.al. (2005) for the time invariant case, in both approaches it is crucial to verify that the delay operator is an admissible observation operator for the translation semigroup (in the sense of Pritchard and Salamon (1985), Salamon (1987), Weiss (1989a)). As a result, one can use the 'Lebesgue extension' of the delay operator with respect to the shift semigroup, as defined by Weiss (1989a). It turns out that the Lebesgue extension greatly simplifies the manipulation of variation of constants formulas, see Hadd and Idrissi (2005), Hadd et.al. (2005). We will also work with Lebesgue extensions, see e.g. Proposition 3.5, which have been introduced and studied in Schnaubelt (2002b) for the time varying case.

But there occurs an unexpected problem. In the time invariant case one can allow for all delay operators given by kernels having bounded variation, see Bátkai and Piazzera (2005), Bensoussan et.al. (1992), or Example 3.2. In the time varying case this is not possible as shown by Example 3.6: The solution of the scalar equation

$$
x^{\prime}(t)=x(t-\rho(t)), \quad t \geq 0, \quad x(t)=f(t), \quad-1 \leq t \leq 0,
$$

does not depend continuously in 2 -norm on $f$ if, say, $\rho(t)=t+1 / 4$ for $1 / 4 \leq t \leq 1 / 2$. We introduce in Section 3 the rather general condition $(\mathrm{H})$ on the kernels $k(t, \theta)$ which yields well-posedness of the delay equation. In the above example one has to assume that $\rho^{\prime}$ is strictly smaller than 1, see Example 3.3. 
There is another, maybe deeper difference to the time invariant theory. In the time invariant case one has the generation theory for semigroups, the existence theory for inhomogeneous equations, and the so-called extrapolation space of the semigroup which allows to treat unbounded control operators, see Weiss (1989b). These concepts and results do not exist in this generality for time varying systems (with the partial exception of parabolic equations, see Acquistapace and Terreni (1999) and Example 5.2). If one wants to develop a general theory it thus seems to be advisable to work with an integrated version of (nLDS), see (3.2) and (5.1), as it was done in Schnaubelt (2002b). As a consequence, in general one does not have the full representation and regularity theory known from time invariant problems, see Section 2. Control theoretic properties are not affected by this reformulation if they are expressed in terms of the input, output or input-output maps, cf. Section 5 of Schnaubelt (2002b).

For the reader's convenience we briefly recall the relevant background from Schnaubelt (2002b) and related works and introduce (much of) our notation in Section 2. Section 3 deals with retarded time varying evolution equations. In Proposition 3.5 we establish new variation of constants formulas for delay equations which are crucial for our approach. Then we construct the regular system corresponding to (nLDS) in Section 4, see Theorem 4.5. Here the main difficulty arises from the fact that we have no 'generators' and thus we can not use generation results as in the time invariant case. Instead, we directly define the operators determining the retarded regular system and then show that they have the desired properties using the results of Sections 2 and 3. As an application, we investigate in the last section the feedback problem corresponding to (nLDS). Under a mild condition on the feedbacks $\Delta(t): Y \rightarrow U$ (also needed in the time invariant case), we prove that the closed loop system is again a regular well-posed system, and we establish various equations relating the open and the closed loop system, see Theorem 5.1. Finally, in Example 5.2 we treat a feedback problem associated to a time varying parabolic boundary control problem with input and state delays.

\section{TIME VARYING REGULAR SYSTEMS}

In this section, we recall several definitions and results on time varying control problems taken from Schnaubelt (2002b). Throughout, $X, Y$, and $U$ denote Banach spaces. We endow $L_{l o c}^{2}(J, Z)$ with its usual Fréchet topology, where $J \subset \mathbb{R}$ is a closed interval and $Z$ is a Banach space. The letter $c$ denotes a generic constant. An evolution family on $X$ is a set $T=(T(t, s))_{t \geq s \geq 0} \subset \mathcal{L}(X)$ (the space of bounded linear operators on $X$ ) such that

(i) $T(t, s)=T(t, r) T(r, s), T(s, s)=I$,

(ii) $(t, s) \mapsto T(t, s)$ is strongly continuous, and

(iii) $\|T(t, s)\| \leq M e^{\omega(t-s)}$

for all $t \geq r \geq s \geq 0$ and constants $M \geq 1, \omega \in \mathbb{R}$. For an evolution family $T$, we set

$$
\left(\mathbb{K}_{s}^{T} f\right)(t):=\int_{s}^{t} T(t, \tau) f(\tau) d \tau
$$

for all $t \geq s \geq 0$ and $f \in L_{l o c}^{2}([s, \infty), X)$. 
Evolution families arise as solution operators of time varying evolution equations

$$
w^{\prime}(t)=A(t) w(t), \quad t \geq s, \quad w(s)=x,
$$

where $A(t), t \geq 0$, are linear operators on $X$ with domains $D(A(t))$. A solution of $(2.1)$ is a function $w \in C^{1}([s, \infty), X)$ such that $w(t) \in D(A(t))$ for $t \geq s$ and (2.1) holds. We say that $(2.1)$ is well-posed, or that $A(\cdot)$ generate $T$, if there is an evolution family $T$ on $X$ such $T(t, s) D(A(s)) \subset D(A(t))$ for $t \geq s$ and $w(t)=T(t, s) x$ is the unique solution of (2.1) for each $x \in D(A(s))$. However, there are evolution families which do not solve an evolution equation (e.g., $T(t, s)=q(t) / q(s)$ on $X=\mathbb{C}$ where $q$ is a continuous, nondifferentiable function). In fact, in contrast to the time invariant semigroup case, there is no general characterization of well-posedness of (2.1) in terms of the operators $A(t)$. In the present paper (as in Schnaubelt (2002b)) we will work only with evolution families without making the additional assumption that they have generators $A(t)$. In particular, we refer to an underlying evolution equation such as (2.1) or (nLDS) only in order to illustrate our results. More information on time varying Cauchy problems can be found in Chicone and Latushkin (1999), Schnaubelt (2002a), and the references therein.

The pair $(T, \Phi):=(T,\{\Phi(t, s): t \geq s \geq 0\})$ is called a time varying control system (on $U$ and $X)$ if $\Phi(t, s): L_{l o c}^{2}([s, \infty), U) \rightarrow X, t \geq s \geq 0$, are linear operators such that

$$
\begin{aligned}
& \Phi(t, s) u=\Phi(t, r)(u \mid[r, \infty))+T(t, r) \Phi(r, s) u, \quad t \geq r \geq s \geq 0, \\
& \|\Phi(t, s) u\|_{X} \leq \beta\|u\|_{L^{2}([s, t], U)}, \quad 0 \leq s \leq t \leq s+t_{0},
\end{aligned}
$$

for $u \in L_{l o c}^{2}\left(\mathbb{R}_{+}, U\right), t_{0}>0$, and a constant $\beta=\beta\left(t_{0}\right)>0$. Then $t \mapsto \Phi(t, s) u \in X$ is continuous for $t \geq s$ by Proposition 3.5 in Schnaubelt (2002b). Note that $\Phi(s, s) u=0$. In the sequel, we will mostly use the same symbol for a function $u$ and its restrictions.

In the time invariant case, control systems are always given by admissible control operators $B$ due to Weiss $(1989 \mathrm{~b})$. It is not clear whether one can extend this result to the time varying in general, see Schnaubelt (2002b) for a discussion. At least, every time varying control system can be represented by bounded control operators in an approximative sense: Set $B_{n}(t) z:=n \Phi\left(t, t-\frac{1}{n}\right) u_{z}$ for $z \in U, n \in \mathbb{N}$, and $t \geq 0$, where $u_{z}(s):=z$ for $s \in \mathbb{R}$ and $\Phi(t, s) u:=\Phi(t, 0) u$ if $t \geq 0 \geq s$. Then Proposition 3.5 in Schnaubelt (2002b) yields

$$
\Phi(t, s) u=\lim _{n \rightarrow \infty} \int_{s}^{t} T(t, \tau) B_{n}(\tau) u(\tau) d \tau
$$

(in $X$ ) for $u \in L_{l o c}^{2}\left(\mathbb{R}_{+}, U\right)$ and $t \geq s \geq 0$, where the limit is locally uniform in $t$.

Let $\Psi(s): X \longrightarrow L_{l o c}^{2}([s, \infty), Y), s \geq 0$, be linear operators satisfying

$$
\Psi(s) x=\Psi(t) T(t, s) x \quad \text { on }[t, \infty) \quad \text { and } \quad \int_{s}^{s+t_{0}}\|(\Psi(s) x)(t)\|^{2} d t \leq \gamma\|x\|^{2}
$$

for $t \geq s \geq 0, x \in X, t_{0}>0$, and a constant $\gamma=\gamma\left(t_{0}\right)>0$. Then $(T, \Psi):=(T,\{\Psi(s), s \geq$ $0\}$ ) is called a time varying observation system (on $X$ and $Y$ ) for $T$. For linear operators $C(s): D(C(s)) \subseteq X \rightarrow Y, s \geq 0$, we define the set

$$
D_{s}(C(\cdot)):=\left\{f \in L_{l o c}^{2}([s, \infty), X): f(t) \in D(C(t)) \text { for a.e. } t \geq s,\right.
$$




$$
\left.C(\cdot) f(\cdot) \in L_{l o c}^{2}([s, \infty), Y)\right\} .
$$

Let $\underline{X}_{s}$ be dense subspaces of $X$ and $C(s): D(C(s)) \subseteq X \longrightarrow Y, s \geq 0$, be linear operators such that $T(\cdot, s) x \in D_{s}(C(\cdot))$ and

$$
\int_{s}^{s+t_{0}}\|C(t) T(t, s) x\|^{2} d t \leq \gamma\|x\|^{2}
$$

for $t_{0}, s \geq 0, x \in \underline{X}_{s}$, and a constant $\gamma=\gamma\left(t_{0}\right)>0$. Then we say that $C(s), s \geq 0$, are admissible observation operators for $T$. Note that the admissibility of $C(\cdot)$ for $T$ guarantees that the mappings

$$
\Psi(s): D(C(s)) \rightarrow L_{l o c}^{2}([s, \infty), Y), \quad \Psi(s) x:=C(\cdot) T(\cdot, s) x, \quad s \geq 0,
$$

possess unique extensions (again noted by $\Psi(s)$ ) to linear continuous operators from $X$ to $L_{l o c}^{2}([s, \infty), Y)$ which yield a time varying observation system $(T, \Psi)$, see Lemma 2.5 in Schnaubelt (2002b).

Conversely, let $(T, \Psi)$ be a time varying observation system, $s \geq 0$, and $\tau>0$. Following Weiss (1989a), we define

$$
\begin{aligned}
C_{\tau}(s) x & =\frac{1}{\tau} \int_{s}^{s+\tau}(\Psi(s) x)(\sigma) d \sigma, \quad x \in X, \\
D(\widetilde{C}(s)) & :=\left\{x \in X: \lim _{\tau \searrow 0} C_{\tau}(s) x \quad \text { exists in } Y\right\}, \quad \widetilde{C}(s) x:=\lim _{\tau \searrow 0} C_{\tau}(s) x .
\end{aligned}
$$

We note that $C_{\tau}(s) \in \mathcal{L}(X, Y)$ and that $\widetilde{C}(s)$ might be non-closable. We say that $\widetilde{C}(\cdot)$ represent $(T, \Psi)$; or that $\widetilde{C}(t)$ are the Lebesgue extensions of $C(t)$ if $\Psi(s)$ is given by (2.5). In the next proposition we summarize Theorem 2.7 and Lemma 2.9 of Schnaubelt (2002b). In particular it is shown that $\widetilde{C}(s)$ is admissible (with $X=\underline{X}_{s}$ ) and that $\Psi(s)$ is always given by $\widetilde{C}(\cdot)$.

Proposition 2.1. Let $(T, \Psi)$ be a time varying observation system, $s \geq 0$, and $x \in X$. Define $C_{\tau}(t)$ and $\widetilde{C}(t)$ for $\tau>0$ and $t \geq 0$ as in (2.6) and (2.7). Then $T(\cdot, s) x \in D_{s}(\widetilde{C}(\cdot))$ and $\Psi(s) x=\widetilde{C}(\cdot) T(\cdot, s) x$. Moreover, $s \mapsto C_{\tau}(s)$ is strongly continuous and the operators $C_{\tau}(\cdot) T(\cdot, s): X \rightarrow L^{2}\left(\left[s, s+t_{0}\right], Y\right)$ are bounded uniformly in $s \geq 0$ and $\tau>0$ and converge strongly to $\Psi(s)$ as $\tau \searrow 0$, where $t_{0}>0$.

The following result is also taken from Schnaubelt (2002b) (see Proposition 2.11 and its proof). We will use it frequently in this paper.

Proposition 2.2. Let $(T, \Psi)$ be a time varying observation system represented by $\widetilde{C}(t)$. Then $\mathbb{K}_{s}^{T} f \in D_{s}(\widetilde{C}(\cdot))$ and

$$
\left\|C_{\tau}(\cdot) \mathbb{K}_{s}^{T} f\right\|_{L^{2}\left(\left[s, s+t_{0}\right], Y\right)},\left\|\widetilde{C}(\cdot) \mathbb{K}_{s}^{T} f\right\|_{L^{2}\left(\left[s, s+t_{0}\right], Y\right)} \leq c t_{0}^{\frac{1}{2}}\|f\|_{L^{2}\left(\left[s, s+t_{0}\right], X\right)}
$$

for $\tau>0, s \geq 0,0<t_{0} \leq t_{1}, f \in L_{\text {loc }}^{2}\left(\mathbb{R}_{+}, X\right)$, and a constant $c=c\left(t_{1}\right)>0$. Moreover, $C_{\tau}(\cdot) \mathbb{K}_{s}^{T}$ converges strongly to $\widetilde{C}(\cdot) \mathbb{K}_{s}^{T}$ as $\tau \searrow 0$. 
Let $(T, \Phi)$ and $(T, \Psi)$ be time varying control and observation systems. If there are linear operators $\mathbb{F}(s): L_{l o c}^{2}([s, \infty), U) \rightarrow L_{l o c}^{2}([s, \infty), Y)$ satisfying

$$
\begin{aligned}
& \mathbb{F}(s) u=\Psi(t) \Phi(t, s) u+\mathbb{F}(t)(u \mid[t, \infty)) \quad \text { on }[t, \infty), \\
& \|\mathbb{F}(s) u\|_{L^{2}\left(\left[s, s+t_{0}\right], Y\right)} \leq \kappa\|u\|_{L^{2}\left(\left[s, s+t_{0}\right], U\right)}
\end{aligned}
$$

for $u \in L_{l o c}^{2}([s, \infty), U), t \geq s \geq 0, t_{0}>0$, and a constant $\kappa=\kappa\left(t_{0}\right)>0$, then $\Sigma=$ $(T, \Phi, \Psi, \mathbb{F})$ is called a well-posed time varying system (on $U, X$, and $Y$ ) with inputoutput operators $\mathbb{F}(s)$. (See Staffans (2004) and Weiss (1994a) for corresponding notions in the time invariant case.) Observe that $\mathbb{F}(s) u=0$ on $[s, t]$ and $\mathbb{F}(s) u=\mathbb{F}(t)(u \mid[t, \infty))$ on $[t, \infty)$ if $u$ vanishes on $[s, t]$. Hence one can define the restrictions

$$
\mathbb{F}(s) \mid[s, t]=: \mathbb{F}(t, s): L^{2}([s, t], U) \rightarrow L^{2}([s, t], Y), \quad t \geq s \geq 0 .
$$

We need two more definitions to use the results on feedback systems from Schnaubelt (2002b). Analogous concepts and results for the time invariant case can be found in Staffans (2004) and Weiss (1994a, 1994b).

Definition 2.3. A well-posed time varying system $\Sigma=(T, \Phi, \Psi, \mathbb{F}$ ) is called regular (with feedthrough $D=0$ ) if

$$
\lim _{\tau \searrow 0} \frac{1}{\tau} \int_{t}^{t+\tau}\left(\mathbb{F}(t) u_{z}\right)(\sigma) d \sigma=0 \quad(\text { in } Y)
$$

and absolutely regular if

$$
\lim _{\tau \searrow 0} \frac{1}{\tau} \int_{t}^{t+\tau}\left\|\left(\mathbb{F}(t) u_{z}\right)(\sigma)\right\|_{Y}^{2} d \sigma=0
$$

for all $t \geq 0$ and $z \in U$, where $u_{z}(s):=z$ for $s \geq 0$.

If $\Sigma$ is regular, then $\mathbb{F}(s) u \in D_{s}(\tilde{C}(\cdot))$ and $\mathbb{F}(s) u=\tilde{C}(\cdot) \Phi(\cdot, s) u$ for $u \in L_{l o c}^{2}([s, \infty), U)$ and $s \geq 0$ by Theorem 3.11 of Schnaubelt (2002b).

Definition 2.4. Let $\Sigma=(T, \Phi, \Psi, \mathbb{F})$ be a well-posed time varying system. We call $\Delta(\cdot) \in L^{\infty}\left(\mathbb{R}_{+}, \mathcal{L}_{s}(Y, U)\right)$ (the space of essentially bounded and strongly measurable operator functions) an admissible feedback for $\Sigma$ if there exists $t_{0}>0$ such that the operators $I-\mathbb{F}\left(s+t_{0}, s\right) \Delta(\cdot), s \geq 0$, have uniformly bounded inverses on $L^{2}\left(\left[s, s+t_{0}\right], Y\right)$.

If $\Sigma$ is absolutely regular and $\Delta(t)$ are admissible feedback operators for $\Sigma$, then the closed-loop system $\Sigma^{\Delta}$ for $\Sigma$ and $\Delta(\cdot)$ exists, and it is also absolutely regular. Moreover, we have several equations relating the open- and closed loop system. To put the formulas in a concise form, we define the operators $\Psi(t, s) x:=(\Psi(s) x) \mid[s, t]$ and

$$
\Sigma(t, s):=\left(\begin{array}{ll}
T(t, s) & \Phi(t, s) \\
\Psi(t, s) & \mathbb{F}(t, s)
\end{array}\right): X \times L^{2}([s, t], U) \longrightarrow X \times L^{2}([s, t], Y)
$$

for $t \geq s \geq 0$. Then it holds

$$
\Sigma^{\Delta}(t, s)-\Sigma(t, s)=\Sigma(t, s)\left(\begin{array}{cc}
0 & 0 \\
0 & \Delta(\cdot)
\end{array}\right) \Sigma_{7}^{\Delta}(t, s)=\Sigma^{\Delta}(t, s)\left(\begin{array}{cc}
0 & 0 \\
0 & \Delta(\cdot)
\end{array}\right) \Sigma(t, s) .
$$


These facts are shown in Theorem 4.4 and Proposition 5.1 of Schnaubelt (2002b), where one can find further results on the relationship between $\Sigma$ and $\Sigma^{\Delta}$. It seems that one needs the stronger condition of absolute regularity to establish such formulas and to obtain (absolute) regularity for the closed loop system (in contrast to the time invariant case).

\section{TIME VARYING DELAY EQUATIONS}

In this section we consider the problem (nLDS) without the control terms and the observation equation, but with an inhomogeneity $h$, i.e., the equations

$$
\begin{aligned}
& w^{\prime}(t)=A(t) w(t)+K(t) w_{t}+h(t), \quad t \geq s \geq 0, \\
& w(s)=x, \quad w_{s}=f .
\end{aligned}
$$

Here $h \in L_{l o c}^{2}\left(\mathbb{R}_{+}, X\right), x \in X$, and $f \in E:=C([-1,0], X)$ are given, and $w_{t}$ is defined by $w_{t}(\theta)=w(t+\theta)$ for $\theta \in[-1,0]$. At first, we assume that the initial data satisfy $x=f(0)$ and that the linear delay operators $K(t): E \rightarrow X, t \geq 0$, are uniformly bounded and strongly measurable in $t$. We concentrate on mild solutions of (3.1), i.e., we are looking for $w \in C([s-1, \infty), X)$ such that

$$
\begin{aligned}
w(t) & =T(t, s) x+\int_{s}^{t} T(t, \tau)\left(K(\tau) w_{\tau}+h(\tau)\right) d \tau, \quad t \geq s \geq 0, \\
w(s+\theta) & =f(\theta), \quad-1 \leq \theta \leq 0,
\end{aligned}
$$

where $A(t), t \geq 0$, generate the evolution family $T(t, s), t \geq s \geq 0$, on $X$. In fact we will investigate (3.2) without assuming that $T$ solves a well-posed Cauchy problem. It is easy to solve (3.2) by a fixed point argument. This gives rise to an evolution family $V(t, s) f:=w_{t}$ on $E$ solving (3.2) with $h=0$. (See e.g. Schnaubelt (2004) for more details and also for differentiability properties of mild solutions.)

However, from the perspective of control theory it is necessary to extend the evolution family $V$ to $L^{2}([-1,0], X)$ (more precisely to $X \times L^{2}([-1,0], X)$, see below). In the time invariant case this can be done in great generality, see e.g. Bátkai and Piazzera (2005), Bensousssan et.al. (1992), or Example 3.2. But in the time varying case, Example 3.6 shows that this extension requires an additional assumption. Lemma 3.1 below is the crucial step in the extension of the evolution family. Before we can state it, we have to introduce some notations.

For a Banach space $Z$, we denote by $B V([-1,0], Z)$ the space of all functions $k$ : $[-1,0] \rightarrow Z$ of bounded variation, i.e. the total variation of $k$

$$
\operatorname{Var}(k)_{-1}^{0}:=\sup \left\{\sum_{j=1}^{n}\left\|k\left(\theta_{j}\right)-k\left(\theta_{j-1}\right)\right\|: 0=\theta_{0}>\theta_{1} \ldots>\theta_{n}=-1, n \in \mathbb{N}\right\}
$$

is finite. Elements of $B V([-1,0], Z)$ are normalized throughout this paper by the requirements $k(-1)=0$ and that $k(\cdot)$ is left-continuous on $[-1,0]$. Hence, extending $k \in B V([-1,0], Z)$ by 0 to $(-\infty, 0], k(\cdot)$ can be also considered as an element of 
$B V((-\infty, 0], Z)$. (This space is defined in the same way as $B V([-1,0], Z)$.) We study delay operators of the form

$$
K(t) f=\int_{-1}^{0} d k(t, \theta) f(\theta), \quad f \in C([-1,0], X), t \geq 0,
$$

satisfying the following assumptions.

(H) The function $\mathbb{R}_{+} \times[-1,0] \ni(t, \theta) \mapsto k(t, \theta) \in \mathcal{L}(Z, X)$ is strongly measurable and $k(t, \cdot) \in B V([-1,0], \mathcal{L}(Z, X))$ with total variation $\eta_{k}(t, \cdot)$. There are constants $c_{k}$ and $c_{k}^{\prime}$ such that $\left\|\eta_{k}(t, \cdot)\right\|:=\operatorname{Var}(k(t, \cdot))_{-1}^{0} \leq c_{k}$ for all $t \geq 0$ and

$$
\int_{0}^{\alpha}\left\|k\left(s+t, \theta^{\prime}-t\right)-k(s+t, \theta-t)\right\| d t \leq c_{k}^{\prime}\left|\theta^{\prime}-\theta\right|
$$

for $\theta^{\prime}, \theta \in[-1,0], s \geq 0$, and some $0<\alpha \leq 1$.

The left translation semigroup $S_{Z}:=\left(S_{Z}(t)\right)_{t \geq 0}$ on $L^{2}([-1,0], Z)$ (endowed with the usual norm $\|\cdot\|_{2}$ ) is defined by

$$
\left(S_{Z}(t) f\right)(\theta):= \begin{cases}f(t+\theta), & t+\theta \leq 0, \\ 0, & t+\theta>0,\end{cases}
$$

for $t \geq 0,-1 \leq \theta \leq 0$, and $f \in L^{2}([-1,0], Z)$. We also set $a \vee b:=\max \{a, b\}$ for $a, b \in \mathbb{R}$.

Lemma 3.1. Assume that $(H)$ holds with $X=Z$. Then we have

$$
\int_{0}^{\alpha}\left\|K(s+t) S_{X}(t) f\right\|^{2} d t \leq c\|f\|_{2}^{2}
$$

for $f \in C([-1,0], X)$ with $f(0)=0,0<\alpha \leq 1, s \geq 0$, and a constant $c>0$. Thus there exist the Lebesgue extensions $\tilde{K}(t), t \geq 0$, of $K(t)$ with respect to $S_{X}$.

Proof. Let $s \geq 0,0<\alpha \leq 1$, and $f \in C([-1,0], X)$ with $f(0)=0$. Set $\sigma_{j}=(j-n) / n$ for $n \in \mathbb{N}$ and $j \in\{0, \cdots, n\}$. For each fixed $t \in[0,1]$, we have

$$
\begin{aligned}
K(s+t) S_{X}(t) f & =\int_{-1}^{-t} d k(s+t, \theta) f(\theta+t) \\
& =\lim _{n \rightarrow \infty} \sum_{j=1}^{n}\left[k\left(t+s, \sigma_{j}-t\right)-k\left(t+s, \sigma_{j-1}-t\right)\right] f\left(\sigma_{j}\right) .
\end{aligned}
$$

(Recall that $k(t, \theta)=0$ if $\theta \leq-1$.) We set $\Lambda_{j}(t, s)=k\left(t+s, \sigma_{j}-t\right)-k\left(t+s, \sigma_{j-1}-t\right)$ for $t, s \geq 0$ and $j=0,1 \ldots, n$. Fatou's lemma, the Cauchy-Schwarz inequality and $(\mathrm{H})$ then imply that

$$
\begin{aligned}
\int_{0}^{\alpha}\left\|K(s+t) S_{X}(t) f\right\|^{2} d t & \leq \liminf _{n \rightarrow \infty} \int_{0}^{\alpha}\left\|\sum_{j=1}^{n} \Lambda_{j}(t, s) f\left(\sigma_{j}\right)\right\|^{2} d t \\
& \leq \liminf _{n \rightarrow \infty} \int_{0}^{\alpha} \sum_{i=1}^{n}\left\|\Lambda_{i}(t, s)\right\| \sum_{j=1}^{n}\left\|\Lambda_{j}(t, s)\right\|\left\|f\left(\sigma_{j}\right)\right\|^{2} d t
\end{aligned}
$$




$$
\begin{aligned}
& \leq c_{k} \liminf _{n \rightarrow \infty} \sum_{j=1}^{n}\left\|f\left(\sigma_{j}\right)\right\|^{2} \int_{0}^{\alpha}\left\|\Lambda_{j}(t, s)\right\| d t \\
& \leq c_{k} c_{k}^{\prime} \lim _{n \rightarrow \infty} \sum_{j=1}^{n}\left\|f\left(\sigma_{j}\right)\right\|^{2}\left|\sigma_{j}-\sigma_{j-1}\right| \\
& =c_{k} c_{k}^{\prime} \int_{-1}^{0}\|f(\sigma)\|^{2} d \sigma .
\end{aligned}
$$

The next example indicates that for time-independent kernels $k$ assumption (H) always holds. The second example shows that a time depending delay $f(-\rho(t))$ is admissible if $\rho^{\prime}$ is strictly smaller than 1, see also Example 3.6.

Example 3.2. Let $k_{0}(\cdot) \in B V([-1,0], \mathcal{L}(X))$ and $k_{1}(\cdot, \cdot): \mathbb{R}_{+} \times[-1,0] \rightarrow \mathcal{L}(X)$ be strongly measurable such that $k_{1}\left(\cdot, \theta_{0}\right)$ is bounded for some $\theta_{0} \in[-1,0]$ and $\left\|k_{1}(t, \cdot)\right\|_{\text {Lip }} \leq$ $c_{1}$ for all $t \geq 0$, where $\|\cdot\|_{\text {Lip }}$ is the Lipschitz norm. We set

$$
k(t, \theta):=k_{1}(t, \theta) k_{0}(\theta), \quad(t, \theta) \in \mathbb{R}_{+} \times[-1,0] .
$$

We claim that $k(\cdot, \cdot)$ satisfies the condition $(\mathrm{H})$. In fact, let $\eta_{0}(\tau)=\operatorname{Var}\left(k_{0}\right)_{-1}^{\tau}$. Then $\eta_{0}$ is nondecreasing and $\left\|k_{0}(\tau)-k_{0}(\sigma)\right\| \leq \eta_{0}(\tau)-\eta_{0}(\sigma)$ for all $\sigma \leq \tau \leq 0$. For $0<\alpha \leq 1$, $-1 \leq \theta \leq \theta^{\prime} \leq 0$, and $s \geq 0$, we further obtain

$$
\begin{aligned}
& \int_{0}^{\alpha}\left\|k\left(s+t, \theta^{\prime}-t\right)-k(s+t, \theta-t)\right\| d t \\
& \leq \int_{0}^{\alpha}\left\|k_{1}\left(s+t, \theta^{\prime}-t\right)-k_{1}(s+t, \theta-t)\right\|\left\|k_{0}\left(\theta^{\prime}-t\right)\right\| d t \\
& \quad \quad+\int_{0}^{\alpha}\left\|k_{1}(s+t, \theta-t)\right\|\left\|k_{0}\left(\theta^{\prime}-t\right)-k_{0}(\theta-t)\right\| d t \\
& \quad \leq c \alpha\left|\theta^{\prime}-\theta\right|+c \int_{0}^{\infty}\left(\eta_{0}\left(\theta^{\prime}-t\right)-\eta_{0}(\theta-t)\right) d t=c \alpha\left|\theta^{\prime}-\theta\right|+c \int_{\theta}^{\theta^{\prime}} \eta_{0}(\tau) d \tau \\
& \leq c\left(\alpha+\eta_{0}(0)\right)\left|\theta^{\prime}-\theta\right|
\end{aligned}
$$

for some constants $c>0$.

Example 3.3. Let $k_{1}(\cdot, \cdot)$ be as in Example 3.2 and assume furthermore that $k_{1}(t,-1)=0$ for all $t \geq 0$. Let $\rho \in C^{1}\left(\mathbb{R}_{+}\right)$such that $\rho^{\prime}(t) \leq 1-\delta$ for $t \geq 0$ and some $\delta>0$. We set

$$
k(t, \theta):=\left\{\begin{array}{ll}
k_{1}(t, \theta), & \theta \geq-\rho(t), \\
0, & \theta<-\rho(t),
\end{array} \quad \text { and } \quad I(s, \theta):=\{t \in[0, \alpha]: t-\rho(s+t) \leq \theta\} .\right.
$$

for $(t, \theta) \in \mathbb{R}_{+} \times[-1,0]$, and $0<\alpha \leq 1$. Then $k(\cdot, \cdot)$ satisfies $(\mathrm{H})$. Indeed, since $k_{1}(t,-1)=$ 0 for all $t \geq 0$, one can see that $k(t, \cdot) \in B V([-1,0], \mathcal{L}(X))$ and $\operatorname{Var}(k(t, \cdot))_{-1}^{0} \leq c_{1}$ for all $t \geq 0$. On the other hand, let $\lambda$ be the Lebesgue measure and $-1 \leq \theta<\theta^{\prime} \leq 0$. Observe that the function $\varphi_{s}(t)=t-\rho(s+t)$ strictly increases and that $\left\|\varphi_{s}^{-1}\right\|_{L i p} \leq \delta^{-1}$. Thus 
we can estimate

$$
\begin{aligned}
\int_{0}^{\alpha} \| k(s & \left.+t, \theta^{\prime}-t\right)-k(s+t, \theta-t) \| d t \\
& =\int_{I(s, \theta)}\left\|k_{1}\left(s+t, \theta^{\prime}-t\right)-k_{1}(s+t, \theta-t)\right\| d t+\int_{I\left(s, \theta^{\prime}\right) \backslash I(s, \theta)}\left\|k_{1}\left(s+t, \theta^{\prime}-t\right)\right\| d t \\
& \leq c \alpha\left|\theta^{\prime}-\theta\right|+c \lambda\left\{t \in[0, \alpha], \theta \leq \varphi_{s}(t) \leq \theta^{\prime}\right\} \\
& \leq c \alpha\left|\theta^{\prime}-\theta\right|+c\left|\varphi_{s}^{-1}\left(\theta^{\prime}\right)-\varphi_{s}^{-1}(\theta)\right| \leq c\left(\delta^{-1}+\alpha\right)\left|\theta^{\prime}-\theta\right| .
\end{aligned}
$$

As mentioned above, we want to solve (3.2) in an $L^{2}$-setting. To this purpose, we introduce the Banach space

$$
\mathcal{X}_{0}:=X \times L^{2}([-1,0], X) \quad \text { with the norm } \quad\left\|\left(\begin{array}{l}
x \\
f
\end{array}\right)\right\|_{\mathcal{X}_{0}}^{2}:=\|x\|^{2}+\|f\|_{2}^{2}
$$

and the operators

$$
\begin{aligned}
\mathcal{T}(t, s) & :=\left(\begin{array}{cc}
T(t, s) & 0 \\
T_{t, s} & S_{X}(t-s)
\end{array}\right), \quad t \geq s \geq 0, \\
\left(T_{t, s} x\right)(\theta) & := \begin{cases}T(t+\theta, s) x, & s-t<\theta \leq 0, \\
0, & -1 \leq \theta \leq s-t,\end{cases}
\end{aligned}
$$

for $\theta \in[-1,0], x \in X$, and $t \geq s \geq 0$. It is straightforward to check that $(\mathcal{T}(t, s))_{t \geq s \geq 0}$ is an evolution family on $\mathcal{X}_{0}$. We further define

$$
\begin{aligned}
\mathcal{D}_{0} & :=\left\{\left(\begin{array}{l}
x \\
f
\end{array}\right) \in X \times C([-1,0], X): f(0)=x\right\}, \\
\mathcal{K}(t) & :=\left(\begin{array}{cc}
0 & K(t) \\
0 & 0
\end{array}\right) \quad \text { with } \quad D(\mathcal{K}(t)):=\mathcal{D}_{0}, \quad t \geq 0 .
\end{aligned}
$$

Observe that $\mathcal{T}(t, s)$ yields also an evolution family on $\mathcal{D}_{0}$, which is a Banach space if endowed the norm $\|x\|+\|f\|_{\infty}$. We further set

$$
(\mathbf{1} \otimes x)(\theta)=x \quad \text { for } x \in X \text { and } \theta \in[-1,0] .
$$

Lemma 3.1 now implies a crucial admissibility property of $\mathcal{K}(t)$.

Lemma 3.4. Assume that $T(\cdot, \cdot)$ is an evolution family on $X$ and that $k(\cdot, \cdot)$ satisfies $(H)$ with $Z=X$. Then $\mathcal{K}(t)$ are admissible observation operators for $\mathcal{T}(\cdot, \cdot)$ on $\mathcal{X}_{0}$. So there exist the Lebesgue extensions $\tilde{\mathcal{K}}(t), t \geq 0$, of $\mathcal{K}(t)$ with respect to $\mathcal{T}(\cdot, \cdot)$. Moreover,

$$
\tilde{\mathcal{K}}(t)\left(\begin{array}{l}
x \\
f
\end{array}\right) \in X \times\{0\} \quad \text { for } \quad\left(\begin{array}{l}
x \\
f
\end{array}\right) \in D(\tilde{\mathcal{K}}(t)), t \geq 0
$$

Proof. Let $s \geq 0, \alpha \in(0,1]$, and $\left(\begin{array}{l}x \\ f\end{array}\right) \in \mathcal{D}_{0}$. Condition $(\mathrm{H})$ and Lemma 3.1 imply that

$$
\begin{aligned}
& \int_{s}^{s+\alpha}\left\|\mathcal{K}(t) \mathcal{T}(t, s)\left(\begin{array}{l}
x \\
f
\end{array}\right)\right\|^{2} d t=\int_{s}^{s+\alpha}\left\|K(t)\left(T_{t, s} x+S_{X}(t-s) f\right)\right\|^{2} d t \\
& =\int_{s}^{s+\alpha}\left\|K(t)\left[T_{t, s} x+S_{X}(t-s)(\mathbf{1} \otimes x)\right]+K(t) S_{X}(t-s)[f-(\mathbf{1} \otimes x)]\right\|^{2} d t \\
& \leq c c_{k}^{2} \int_{s}^{s+\alpha}\left\|T_{t, s} x+S_{X}(t-s)(\mathbf{1} \otimes x)\right\|_{\infty}^{2} d t+c \int_{s}^{s+\alpha}\left\|K(t) S_{X}(t-s)[f-(\mathbf{1} \otimes x)]\right\|^{2} d t
\end{aligned}
$$




$$
\leq c c_{k}^{2} \alpha\|x\|^{2}+c\|f-(\mathbf{1} \otimes x)\|_{2}^{2} \leq c\left(\|x\|^{2}+\|f\|_{2}^{2}\right)
$$

for constants $c>0$. The (easier) case $\alpha>1$ is treated similarly.

Since $\mathcal{K}(t) \mathcal{T}(t, s)\left(\begin{array}{l}x \\ f\end{array}\right) \in X \times\{0\}$ for $\left(\begin{array}{l}x \\ f\end{array}\right) \in \mathcal{D}_{0}$ and $t \geq s \geq 0$, we deduce by approximation that $\tilde{\mathcal{K}}(\cdot) \mathcal{T}(\cdot, s)\left(\begin{array}{c}x \\ f\end{array}\right) \in L_{l o c}^{2}([s, \infty), X \times\{0\})$ for all $\left(\begin{array}{c}x \\ f\end{array}\right) \in \mathcal{X}_{0}$ and $s \geq 0$. Thus $\tilde{\mathcal{K}}(s)\left(\begin{array}{l}x \\ f\end{array}\right) \in X \times\{0\}$ for $\left(\begin{array}{l}x \\ f\end{array}\right) \in D(\tilde{\mathcal{K}}(s))$ by the definition of the Lebesgue extension.

Proposition 3.5. Assume that $T(\cdot, \cdot)$ is an evolution family on $X$ and that $k(\cdot, \cdot)$ satisfies (H) with $Z=X$. Then the following assertions hold.

(a) There is a unique evolution family $\mathcal{T}_{K}(\cdot, \cdot)$ on $\mathcal{X}_{0}$ such that $\tilde{\mathcal{K}}(t)$ are admissible observation operators for $\mathcal{T}_{K}$ and

$$
\begin{aligned}
& \mathcal{T}_{K}(t, s)\left(\begin{array}{l}
x \\
f
\end{array}\right)=\mathcal{T}(t, s)\left(\begin{array}{l}
x \\
f
\end{array}\right)+\int_{s}^{t} \mathcal{T}(t, \tau) \widetilde{\mathcal{K}}(\tau) \mathcal{T}_{K}(\tau, s)\left(\begin{array}{l}
x \\
f
\end{array}\right) d \tau, \\
& \mathcal{T}_{K}(t, s)\left(\begin{array}{l}
x \\
f
\end{array}\right)=\mathcal{T}(t, s)\left(\begin{array}{l}
x \\
f
\end{array}\right)+\int_{s}^{t} \mathcal{T}_{K}(t, \tau) \widetilde{\mathcal{K}}(\tau) \mathcal{T}(\tau, s)\left(\begin{array}{l}
x \\
f
\end{array}\right) d \tau
\end{aligned}
$$

for all $\left(\begin{array}{l}x \\ f\end{array}\right) \in \mathcal{X}_{0}$ and $t \geq s \geq 0$. The operators $\widetilde{\mathcal{K}}(\cdot)$ represent also the observation system given by $\widetilde{\mathcal{K}}(\cdot) \mathcal{T}_{K}(\cdot, s)$ and $\mathcal{T}_{K}$ on $\mathcal{X}_{0}$.

(b) Moreover, $\mathcal{T}_{K}(t, s)$ leaves $\mathcal{D}_{0}$ invariant and yields an evolution family on $\mathcal{D}_{0}$, too. Therefore we can replace $\widetilde{\mathcal{K}}(\tau)$ by $\mathcal{K}(\tau)$ in (3.5) and (3.6) if $\left(\begin{array}{l}x \\ f\end{array}\right) \in \mathcal{D}_{0}$. If we set $\left(\begin{array}{c}v(t) \\ w(t)\end{array}\right)=$ $\mathcal{T}_{K}(t, s)\left(\begin{array}{l}x \\ f\end{array}\right)$ for $t \geq s$ and $v(t)=f(t-s)$ for $s-1 \leq t \leq s$ and $\left(\begin{array}{l}x \\ f\end{array}\right) \in \mathcal{X}_{0}$, then $w(t)=v_{t}$. (c) If $\left(\begin{array}{l}x \\ f\end{array}\right) \in \mathcal{D}_{0}, h \in L_{\text {loc }}^{2}\left(\mathbb{R}_{+}, X\right)$, and $s \geq 0$, then the unique mild solution $v \in C([s-$ $1, \infty), X)$ of $(3.2)$ is given by $v(t)=f(t-s)$ for $s-1 \leq t \leq s$ and

$$
\left(\begin{array}{c}
v(t) \\
v_{t}
\end{array}\right)=\mathcal{T}_{K}(t, s)\left(\begin{array}{c}
x \\
f
\end{array}\right)+\int_{s}^{t} \mathcal{T}_{K}(t, \tau)\left(\begin{array}{c}
h(\tau) \\
0
\end{array}\right) d \tau .
$$

Proof. (a)We define $\Psi^{0}(s): \mathcal{D}_{0} \rightarrow \mathcal{X}_{0}$ by setting $\Psi^{0}(s)\left(\begin{array}{l}x \\ f\end{array}\right):=\mathcal{K}(\cdot) \mathcal{T}(\cdot, s)\left(\begin{array}{l}x \\ f\end{array}\right)$. Lemma 3.4 shows that $\left(\mathcal{T}, \Psi^{0}\right)$ can be extended to a time varying observation system on $\mathcal{X}_{0}$ with observation space $\mathcal{X}_{0}$. We further set $\Phi^{0}(\cdot, s) u:=\mathbb{K}_{s}^{\mathcal{T}} u$ and $\mathbb{F}^{0}(s) u=\widetilde{\mathcal{K}}(\cdot) \mathbb{K}_{s}^{\mathcal{T}} u$ for $s \geq 0$ and $u \in L_{l o c}^{2}\left([s, \infty), \mathcal{X}_{0}\right)$. The system $\left(\mathcal{T}, \Phi^{0}, \Psi^{0}, \mathbb{F}^{0}\right)$ is absolutely regular as observed in Remark 4.6(a) in Schnaubelt (2002b). The feedback $I_{\mathcal{X}_{0}}$ is admissible since $\left\|\mathbb{F}^{0}\left(s+t_{0}, s\right)\right\|_{2} \leq c t_{0}^{1 / 2} \leq \frac{1}{2}$ for $s \geq 0$ due to Proposition 2.2, if we take a sufficiently small $t_{0}>0$. Then part (a) of the assertion follows from Theorem 4.5 in Schnaubelt (2002b), except for the last claim. To establish this claim, we estimate

$\left\|\frac{1}{r} \int_{s}^{s+r} \widetilde{\mathcal{K}}(t) \int_{s}^{t} \mathcal{T}(t, \tau) \widetilde{\mathcal{K}}(\tau) \mathcal{T}_{K}(\tau, s)\left(\begin{array}{l}x \\ f\end{array}\right) d \tau d t\right\| \leq c r^{-1} r^{\frac{1}{2}} r^{\frac{1}{2}}\left\|\widetilde{\mathcal{K}}(\cdot) \mathcal{T}_{K}(\cdot, s)\left(\begin{array}{l}x \\ f\end{array}\right)\right\|_{L^{2}\left([s, s+r], \mathcal{X}_{0}\right)}$

for $s \geq 0, r>0$, and $\left(\begin{array}{l}x \\ f\end{array}\right) \in \mathcal{X}_{0}$, using Hölder's inequality and Proposition 2.2. The right hand side of this inequality tends to 0 as $r \rightarrow 0$. Equation (3.5) thus implies that the observation systems $\left(\mathcal{T}, \Psi^{0}(s)\right)$ and $\left(\mathcal{T}_{K}, \widetilde{\mathcal{K}}(\cdot) \mathcal{T}_{K}(\cdot, s)\right)$ are represented by the same operators, namely $\widetilde{\mathcal{K}}(\cdot)$. 
(b) Due to (3.4), the first and second component of the integral in (3.5) are equal to

$$
\int_{s}^{t} T(t, \tau)\left[\widetilde{\mathcal{K}}(\tau) \mathcal{T}_{K}(\tau, s)\left(\begin{array}{l}
x \\
f
\end{array}\right)\right]_{1} d \tau \quad \text { and } \quad \int_{s}^{(t+\theta) \vee s} T(t+\theta, \tau)\left[\widetilde{\mathcal{K}}(\tau) \mathcal{T}_{K}(\tau, s)\left(\begin{array}{l}
x \\
f
\end{array}\right)\right]_{1} d \tau
$$

respectively, where $\left(\begin{array}{l}x \\ f\end{array}\right) \in \mathcal{X}_{0}, \theta \in[-1,0], t \geq s \geq 0$. As a result, the integral takes values in $\mathcal{D}_{0}$. It is then straightforward to check that $\mathcal{T}_{K}(t, s)$ yields an evolution family on $\mathcal{D}_{0}$. The last assertion in (b) follows from (3.5), (3.3), and (3.8).

(c) Let $\left(\begin{array}{l}x \\ f\end{array}\right) \in \mathcal{D}_{0}, h \in L_{\text {loc }}^{2}\left(\mathbb{R}_{+}, X\right)$, and $s \geq 0$. The uniqueness of mild solutions to (3.2) is a standard consequence of Gronwall's inequality. We denote the right hand side of (3.7) by $\left(\begin{array}{l}v(t) \\ w(t)\end{array}\right)$. Equation (3.5) yields

$$
\begin{aligned}
\left(\begin{array}{c}
v(t) \\
w(t)
\end{array}\right)=\mathcal{T}(t, s)\left(\begin{array}{l}
x \\
f
\end{array}\right)+\int_{s}^{t} \mathcal{T}(t, \tau) \mathcal{K}(\tau) \mathcal{T}_{K}(\tau, s)\left(\begin{array}{l}
x \\
f
\end{array}\right) d \tau+\int_{s}^{t} \mathcal{T}(t, \tau)\left(\begin{array}{c}
h(\tau) \\
0
\end{array}\right) d \tau \\
+\int_{s}^{t} \int_{\tau}^{t} \mathcal{T}(t, \sigma) \widetilde{\mathcal{K}}(\sigma) \mathcal{T}_{K}(\sigma, \tau)\left(\begin{array}{c}
h(\tau) \\
0
\end{array}\right) d \sigma d \tau
\end{aligned}
$$

We denote the double integral in (3.9) by $J$. Employing the bounded operators $\mathcal{K}_{1 / n}(t)$ defined as in (2.6), we rewrite $J$ as

$$
\begin{aligned}
J & =\lim _{n \rightarrow \infty} \int_{s}^{t} \int_{\tau}^{t} \mathcal{T}(t, \sigma) \mathcal{K}_{\frac{1}{n}}(\sigma) \mathcal{T}_{K}(\sigma, \tau)\left(\begin{array}{c}
h(\tau) \\
0
\end{array}\right) d \sigma d \tau \\
& =\lim _{n \rightarrow \infty} \int_{s}^{t} \mathcal{T}(t, \sigma) \mathcal{K}_{\frac{1}{n}}(\sigma) \int_{s}^{\sigma} \mathcal{T}_{K}(\sigma, \tau)\left(\begin{array}{c}
h(\tau) \\
0
\end{array}\right) d \tau d \sigma \\
& =\int_{s}^{t} \mathcal{T}(t, \sigma) \widetilde{\mathcal{K}}(\sigma) \int_{s}^{\sigma} \mathcal{T}_{K}(\sigma, \tau)\left(\begin{array}{c}
h(\tau) \\
0
\end{array}\right) d \tau d \sigma
\end{aligned}
$$

using Propositions 2.1 and 2.2, part (a), and Fubini's theorem. We set $\varphi(t)=\left[\widetilde{\mathcal{K}}(t)\left(\begin{array}{c}v(t) \\ w(t)\end{array}\right)\right]_{1}$. Formulas (3.9), (3.10), (3.7), and (3.3) then imply

$$
\left(\begin{array}{c}
v(t) \\
w(t)
\end{array}\right)=\left(\begin{array}{c}
T(t, s) x \\
T_{t, s} x+S_{X}(t-s) f
\end{array}\right)+\left(\begin{array}{c}
\int_{s}^{t} T(t, \tau)(\varphi(\tau)+h(\tau)) d \tau \\
\int_{s}^{t} T_{t, \tau}(\varphi(\tau)+h(\tau)) d \tau
\end{array}\right) .
$$

If we set $v(t)=f(t-s)$ for $s-1 \leq t \leq s$, we deduce $w(t)=v_{t}$ and thus $\left(\begin{array}{c}v(t) \\ w(t)\end{array}\right) \in \mathcal{D}_{0}$. Hence, $\varphi(t)=K(t) v_{t}$ and $v$ is the mild solution of (3.2).

The following simple example shows that one really needs an extra assumption in Proposition 3.5, cf. Example 3.3.

Example 3.6. On $X=\mathbb{C}$ we consider

$$
x^{\prime}(t)=x(t-\rho(t)), \quad t \geq 0, \quad x(t)=f(t), \quad-1 \leq t \leq 0,
$$

for the time depending delay

$$
\rho(t)= \begin{cases}\frac{1}{2}, & 0 \leq t \leq \frac{1}{4} \\ t+\frac{1}{4}, & \frac{1}{4} \leq t \leq \frac{1}{2} \\ \frac{3}{4}, & t \geq \frac{1}{2}\end{cases}
$$


Suppose that $f$ is continuous and $f(0)=0$. Then,

$$
\begin{aligned}
& x(t)=\int_{0}^{t} f\left(\tau-\frac{1}{2}\right) d \tau=\int_{-\frac{1}{2}}^{t-\frac{1}{2}} f(\tau) d \tau \quad \text { for } \quad 0 \leq t \leq \frac{1}{4}, \\
& x(t)=\int_{-\frac{1}{2}}^{-\frac{1}{4}} f(\tau) d \tau+\left(t-\frac{1}{4}\right) f\left(-\frac{1}{4}\right) \quad \text { for } \quad \frac{1}{4} \leq t \leq \frac{1}{2} .
\end{aligned}
$$

Hence, $x(t)$ does not depend continuously on $f$ in the $L^{2}$-norm, so that $\mathcal{T}_{K}(\cdot, \cdot)$ can not continuously be extended to $\mathcal{X}_{0}$ in this case.

\section{TIME VARYING REGULAR SYSTEMS WITH STATE AND INPUT DELAYS}

In this section we want to show that (nLDS) corresponds to a time varying regular linear system $\Sigma_{K, L}$. The system $\Sigma_{K, L}$ is determined by a regular linear system $(T, \Phi, \Psi, \mathbb{F})$ on the spaces $U, X$, and $Y$ and by the state and input delay operators

$$
K(t) f=\int_{-1}^{0} d k(t, \theta) f(\theta) \quad \text { and } \quad L(t) g=\int_{-1}^{0} d l(t, \theta) g(\theta)
$$

$f \in C([-1,0], X), g \in C([-1,0], U), t \geq 0$, whose kernels $k$ and $l$ satisfy assumption $(\mathrm{H})$ for $X=Z$ and $U=Z$, respectively. The state space of $\Sigma_{K, L}$ is given by

$$
\mathcal{X}:=X \times L^{2}([-1,0], X) \times L^{2}([-1,0], U) \quad \text { with norm } \quad\left\|\left(\begin{array}{l}
x \\
f \\
g
\end{array}\right)\right\|_{\mathcal{X}}^{2}:=\|x\|^{2}+\|f\|_{2}^{2}+\|g\|_{2}^{2} .
$$

Quite often we use the factorization $\mathcal{X}=\mathcal{X}_{0} \times L^{2}([-1,0], U)$. We further set

$$
\begin{aligned}
\mathcal{L}(t) & :=\left(\begin{array}{c}
L(t) \\
0
\end{array}\right): C([-1,0], U) \rightarrow \mathcal{X}_{0} \\
\mathcal{T}_{K, L}(t, s) & :=\left(\begin{array}{cc}
\mathcal{T}_{K}(t, s) & \int_{s}^{t} \mathcal{T}_{K}(t, \tau) \mathcal{L}(\tau) S_{U}(\tau-s) d \tau \\
0 & S_{U}(t-s)
\end{array}\right),
\end{aligned}
$$

for $t \geq s \geq 0$, where $\mathcal{T}_{K, L}(t, s)$ is at first defined on the space

$$
\mathcal{D}:=\left\{(x, f, g) \in \mathcal{D}_{0} \times C([-1,0], U): g(0)=0\right\} \subset \mathcal{X} .
$$

Arguing as in the proof of Proposition 3.5(b), one verifies that the integral in (4.1) takes values in $\mathcal{D}_{0}$. It is then straightforward to show that $\mathcal{T}_{K, L}(\cdot, \cdot)$ is an evolution family on $\mathcal{D}$. Due to Lemma 3.1, there exist the Lebesgue extensions $\widetilde{L}(t), t \geq 0$, of the restriction of $L(t)$ to $C_{0}([-1,0), U)$ with respect to the shift semigroup $S_{U}$. Thus we may define

$$
\widetilde{\mathcal{L}}(t):=\left(\begin{array}{c}
\widetilde{L}(t) \\
0
\end{array}\right): D(\tilde{L}(t)) \subset C_{0}([-1,0), U) \rightarrow \mathcal{X}_{0} .
$$

Observe that Proposition 3.5 and Lemma 3.1 allow to extend $\mathcal{T}_{K, L}(t, s)$ to the operator

$$
\mathcal{T}_{K, L}(t, s)=\left(\begin{array}{cc}
\mathcal{T}_{K}(t, s) & \int_{s}^{t} \mathcal{T}_{K}(t, \tau) \widetilde{\mathcal{L}}(\tau) S_{U}(\tau-s) d \tau \\
0 & S_{U}(t-s)
\end{array}\right)
$$

on $\mathcal{X}, t \geq s \geq 0$, and that $\mathcal{T}_{K, L}$ is an evolution family on $\mathcal{X}$. It will be the evolution family of the system $\Sigma_{K, L}$. The right upper entry of this matrix feeds the initial history $g=u_{s}$ of the input $u$ into the system; the right lower entry shifts $g$ according to the time step from $s$ to $t$. 
We now proceed in three steps: First we add the non retarded observation of the given undelayed system. Then we apply the input delay $L(t)$. Finally, we combine both parts by an input-output operator.

We suppose that $(T, \Psi)$ is a time varying observation system on $X$ and $Y$ with representing operators $\widetilde{C}(t)$ as in (2.7). As a preliminary step, we define

$$
\begin{aligned}
\mathcal{C}(t) & :=\left(\begin{array}{ll}
\widetilde{C}(t) & 0
\end{array}\right) \quad \text { with } \quad D(\mathcal{C}(t)):=D(\widetilde{C}(t)) \times L^{2}([-1,0], X), \quad t \geq 0, \\
\Psi_{K}(s)\left(\begin{array}{l}
x \\
f
\end{array}\right) & :=\mathcal{C}(\cdot) \mathcal{T}_{K}(\cdot, s)\left(\begin{array}{l}
x \\
f
\end{array}\right), \quad\left(\begin{array}{l}
x \\
f
\end{array}\right) \in D(\mathcal{C}(s)), \quad s \geq 0 .
\end{aligned}
$$

Lemma 4.1. Under the above assumptions, the operators $\Psi_{K}(s), s \geq 0$, defined in (4.3) can be extended to a time varying observation system for $\mathcal{T}_{K}$ on $\mathcal{X}_{0}$ and $Y$ which is represented by the operators $\mathcal{C}(t): D(\mathcal{C}(t)) \rightarrow Y$.

Proof. Let $s \geq 0$ and $\left(\begin{array}{l}x \\ f\end{array}\right) \in \mathcal{X}_{0}$. Proposition 3.5 shows that the function $h(\cdot ; s, x, f):=$ $\left[\widetilde{\mathcal{K}}(\cdot) \mathcal{T}_{K}(\cdot, s)\left(\begin{array}{l}x \\ f\end{array}\right)\right]_{1}$ belongs to $L_{l o c}^{2}([s, \infty), X)$ and that

$$
\left[\mathcal{T}_{K}(t, s)\left(\begin{array}{l}
x \\
f
\end{array}\right)\right]_{1}=T(t, s) x+\int_{s}^{t} T(t, \tau) h(\tau ; s, x, f) d \tau
$$

Due to this equality, Propositions 2.1, 2.2, and 3.5 imply that $\mathcal{C}(t), t \geq 0$, are admissible observation operators for the evolution family $\mathcal{T}_{K}(\cdot, \cdot)$. Thus we have established the first assertion. We further deduce from (4.4) that

$$
\frac{1}{\tau} \int_{s}^{s+\tau}\left(\Psi_{K}(s)\left(\begin{array}{l}
x \\
f
\end{array}\right)\right)(t) d t=\frac{1}{\tau} \int_{s}^{s+\tau} \widetilde{C}(t) T(t, s) x d t+\frac{1}{\tau} \int_{s}^{s+\tau} \widetilde{C}(t)\left(\mathbb{K}_{s}^{T} h(\cdot ; s, x, f)\right)(t) d t
$$

for $\tau>0$. The first term on the right side converges as $\tau \searrow 0$ if and only if $x \in D(\widetilde{C}(s))$; and then the limit is equal to $\widetilde{C}(s) x$. Hölder's inequality and Proposition 2.2 further yield

$$
\left\|\frac{1}{\tau} \int_{s}^{s+\tau}\left(\widetilde{C}(\cdot) \mathbb{K}_{s}^{T} h(\cdot ; s, x, f)\right)(t) d t\right\| \leq c\|h(\cdot ; s, x, f)\|_{L^{2}([s, s+\tau], X)} \rightarrow 0
$$

as $\tau \rightarrow 0$. The second assertion follows from these facts.

As a result, Proposition 2.2 yields that

$$
\int_{s}^{\cdot} \mathcal{T}_{K}(\cdot, \tau)\left(\underset{0}{\widetilde{L}(\tau) S_{U}(\tau-s) g}\right) d \tau \in D_{s}(\mathcal{C}(\cdot))
$$

for all $g \in L^{2}([-1,0], U)$ since $\widetilde{L}(\cdot) S_{U}(\cdot-s) g \in L_{l o c}^{2}([s, \infty), X)$. Thus we can define the operators

$$
\Psi_{K, L}(s)(x, f, g):=\Psi_{K}(s)\left(\begin{array}{l}
x \\
f
\end{array}\right)+\mathcal{C}(\cdot) \mathbb{K}_{s}^{\mathcal{T}_{K}}\left(\begin{array}{c}
\widetilde{L}(\cdot) S_{U}(\cdot-s) g \\
0
\end{array}\right)
$$

for $s \geq 0$ and $(x, f, g) \in \mathcal{X}$.

Proposition 4.2. Assume that $(T, \Psi)$ is a time varying observation system on $X$ and $Y$ with representing operators $\widetilde{C}(t): D(\widetilde{C}(t)) \rightarrow Y$ and that the kernels $k$ and $l$ satisfy assumption (H) for $X=Z$ and $U=Z$, respectively. Then $\left(\mathcal{T}_{k, L}, \Psi_{K, L}\right)$ defined in (4.2) and (4.6) yields a time varying observation system on $\mathcal{X}$ and $Y$ represented by $\mathbf{C}(t):=$ $(\widetilde{C}(t), 0,0)$ with $D(\mathbf{C}(t)):=D(\widetilde{C}(t)) \times L^{2}([-1,0], X) \times L^{2}([-1,0], U), t \geq 0$. 
Proof. Lemma 4.1, Proposition 2.2 and Lemma 3.1 show that the operators $\Psi_{K, L}(s)$ satisfy the estimate in (2.4). Let now $(x, f, g) \in \mathcal{X}$ and $\rho \geq t \geq s \geq 0$. Then

$$
\begin{aligned}
& {\left[\Psi_{K, L}(t) \mathcal{T}_{K, L}(t, s)(x, f, g)\right](\rho)=\left[\Psi_{K}(t) \mathcal{T}_{K}(t, s)\left(\begin{array}{l}
x \\
f
\end{array}\right)\right](\rho)+\mathcal{C}(\rho) \mathcal{T}_{K}(\rho, t)\left[\mathbb{K}_{s}^{\mathcal{T}_{K}}\left(\begin{array}{c}
\widetilde{L}(\cdot) S_{U}(\cdot-s) g \\
0
\end{array}\right)\right](t)} \\
& +\mathcal{C}(\rho)\left[\mathbb{K}_{t}^{\mathcal{T}_{K}}\left(\widetilde{L}(\cdot) S_{U}(\cdot-t) S_{U}(t-s) g\right)\right](\rho) \\
& =\left(\Psi_{K}(s)\left(\begin{array}{l}
x \\
f
\end{array}\right)\right)(\rho)+\mathcal{C}(\rho)\left[\mathbb{K}_{s}^{\mathcal{T}_{K}}\left(\underset{L}{ }(\cdot) S_{U}(\cdot-s) g\right)\right](\rho) \\
& =\left[\Psi_{K, L}(s)(x, f, g)\right](\rho) \text {. }
\end{aligned}
$$

Thus we have shown the first assertion. The representation of the observation system can be computed as in the proof of Lemma 4.1, using Lemma 4.1, Proposition 2.2 and the fact that $\widetilde{L}(\cdot) S_{U}(\cdot-s) g \in L_{l o c}^{2}([s, \infty), X)$.

In the second step, we suppose that $(T, \Phi)$ is a time varying control system on $U$ and $X$. Let $t \geq s \geq 0, \theta \in[-1,0]$, and $u \in L_{l o c}^{2}([s, \infty), U)$. Then we define

$$
\begin{aligned}
\widehat{\Phi}(t, s) u & :=\left(\begin{array}{c}
\Phi(t, s) u \\
\Phi_{t, s} u
\end{array}\right), \quad\left(\Phi_{t, s} u\right)(\theta):= \begin{cases}\Phi(t+\theta, s) u, & t+\theta \geq s \\
0, & t+\theta<s\end{cases} \\
\left(R_{t, s} u\right)(\theta) & := \begin{cases}u(t+\theta), & t+\theta>s \\
0, & t+\theta \leq s .\end{cases}
\end{aligned}
$$

Observe that $\widehat{\Phi}(\tau, s) u \in \mathcal{D}_{0}$. Let $0 \leq s \leq \rho \leq t$ and

$$
u(\tau):= \begin{cases}u_{1}(\tau), & \rho<\tau \leq t \\ u_{0}(\tau), & s \leq \tau \leq \rho,\end{cases}
$$

for given functions $u_{0}, u_{1} \in L_{l o c}^{2}([s, \infty), U)$. Then one easily checks that

$$
R_{t, s} u=R_{t, \rho} u_{1}+S_{U}(t-\rho) R_{\rho, s} u_{0} .
$$

The following result can be proved in the same way as Lemma 3.1.

Lemma 4.3. Assume that $l(\cdot, \cdot)$ satisfies $(H)$ with $U=Z$. Let $u \in C([s, \infty), U)$ and $g \in C([-1,0], U)$ with $u(s)=g(0)$. Then we have

$$
\int_{s}^{s+\alpha}\left\|L(t)\left(R_{t, s} u+S_{U}(t-s) g\right)\right\|^{2} d t \leq c\left(\|u\|_{L^{2}([s, s+\alpha], U)}^{2}+\|g\|_{L^{2}([-1,0], U)}^{2}\right)
$$

for $0<\alpha \leq \alpha_{0}, s \geq 0$, and a constant $c=c\left(\alpha_{0}\right)>0$.

We want to use Lemma 4.3 to define the Lebesgue extensions for $L(t)$ with respect to $R_{t, s}$ and $S_{U}(t)$. To that purpose we first have to identify the relevant evolution family. Let $g \in L^{2}([-1,0], U), u \in L^{2}([s, \infty), U)$, and $s \geq 0$. We set $v(\tau)=u(\tau+s)$ for $\tau \geq 0$ and $v(\tau)=g(\tau)$ for $\tau \in[-1,0)$. We further define $S_{U}^{\infty}(t) h=h(\cdot+t)$ and $P h=h \mid[-1,0]$ for $h \in L^{2}([-1, \infty), U)$ and $t \geq 0$. Clearly,

$$
R_{t, s} u+S_{U}(t-s) g=P S_{U}^{\infty}(t-s) v .
$$


Lemma 4.3 then implies that $L(t) P, t \geq 0$, are admissible observation operators for the evolution family $S_{U}^{\infty}(t-s)$ on $L^{2}([-1, \infty), U)$ with observation space $X$. We denote by $\bar{L}(t)$ the corresponding Lebesgue extensions, and we set

$$
\overline{\mathcal{L}}(t):=\left(\begin{array}{c}
\bar{L}(t) \\
0
\end{array}\right): D(\bar{L}(t)) \subset L^{2}([-1, \infty), U) \rightarrow \mathcal{X}_{0}, \quad t \geq 0 .
$$

We can now define the desired time varying control system:

$$
\begin{aligned}
& \Phi_{K, L}(t, s) u:=\left(\begin{array}{c}
\widehat{\Phi}(t, s) u \\
0
\end{array}\right)+\left(\begin{array}{c}
\int_{s}^{t} \mathcal{T}_{K}(t, \tau) \mathcal{K}(\tau) \widehat{\Phi}(\tau, s) u d \tau \\
0
\end{array}\right)+\left(\begin{array}{c}
\int_{s}^{t} \mathcal{T}_{K}(t, \tau) \mathcal{L}(\tau) R_{\tau, s} u d \tau \\
R_{t, s} u
\end{array}\right) \\
& =\left(\begin{array}{c}
\widehat{\Phi}(t, s) u \\
0
\end{array}\right)+\left(\begin{array}{c}
\int_{s}^{t} \mathcal{T}_{K}(t, \tau) \mathcal{K}(\tau) \widehat{\Phi}(\tau, s) u d \tau \\
0
\end{array}\right)+\left(\begin{array}{c}
\int_{s}^{t} \mathcal{T}_{K}(t, \tau) \overline{\mathcal{L}}(\tau) R_{\tau, s} u d \tau \\
R_{t, s} u
\end{array}\right)
\end{aligned}
$$

for $t \geq s \geq 0$ and $u \in C([s, \infty), U)$ with $u(s)=0$ in the first line and $u \in L_{l o c}^{2}([s, \infty), U)$ in the second line. We discuss this definition after the following result.

Proposition 4.4. Assume that $(T, \Phi)$ is a time varying control system on $U$ and $\mathcal{X}$ and that the kernels $k$ and $l$ satisfy assumption (H) for $X=Z$ and $U=Z$, respectively. Then the pair $\left(\mathcal{T}_{K, L}, \Phi_{K, L}\right)$ defined in (4.2) and (4.11) is a time varying control system on $U$ and $\mathcal{X}$.

Proof. Using the inequality in (2.2) and $(\mathrm{H})$, we estimate

$$
\begin{aligned}
\int_{s}^{t}\|\mathcal{K}(\sigma) \widehat{\Phi}(\sigma, s) u\|^{2} d \sigma & \leq \int_{s}^{t}\left[\int_{(s-\sigma) \vee-1}^{0}\|\Phi(\sigma+\theta, s) u\| d \eta_{k}(\sigma, \theta)\right]^{2} d \sigma \\
& \leq \beta^{2} \int_{s}^{t}\left[\int_{(s-\sigma) \vee-1}^{0}\|u\|_{L^{2}([s, t], U)} d \eta_{k}(\sigma, \theta)\right]^{2} d \sigma \\
& \leq \beta^{2} t_{0} c_{k}^{2}\|u\|_{L^{2}([s, t], U)}^{2}
\end{aligned}
$$

for $0 \leq s \leq t \leq s+t_{0}$, and $u \in L_{l o c}^{2}([s, \infty), U)$. Inequality (4.12) and Lemma 4.3 imply that $\Phi_{K, L}(t, s)$ satisfies the estimate in $(2.2)$. Let $0 \leq s \leq \rho \leq t, u_{0} \in C([s, \infty), X)$ with $u_{0}(s)=0$, and $u_{1} \in C([\rho, \infty), X)$ with $u_{0}(\rho)=u_{1}(\rho)=0$. We define the continuous function $u$ as in (4.7). Then we obtain

$$
\begin{aligned}
\widehat{\Phi}(t, s) u & =\left(\begin{array}{c}
\Phi(t, \rho) u_{1}+T(t, \rho) \Phi(\rho, s) u_{0} \\
\Phi_{t, \rho} u_{1}+S_{X}(t-\rho) \Phi_{\rho, s} u_{0}+T_{t, \rho} \Phi(\rho, s) u_{0}
\end{array}\right) \\
& =\mathcal{T}(t, \rho) \widehat{\Phi}(\rho, s) u_{0}+\widehat{\Phi}(t, \rho) u_{1} .
\end{aligned}
$$

Hence (4.8) and (3.6) imply that

$$
\begin{aligned}
\Phi_{K, L}(t, s) u=\left(\begin{array}{c}
\mathcal{T}(t, \rho) \widehat{\Phi}(\rho, s) u_{0}+\widehat{\Phi}(t, \rho) u_{1} \\
0
\end{array}\right) \\
+\left(\begin{array}{c}
\int_{\rho}^{t} \mathcal{T}_{K}(t, \tau) \mathcal{K}(\tau)\left[\widehat{\Phi}(\tau, \rho) u_{1}+\mathcal{T}(\tau, \rho) \widehat{\Phi}(\rho, s) u_{0}\right] d \tau \\
0 \\
17
\end{array}\right)
\end{aligned}
$$




$$
\begin{aligned}
+ & \left(\begin{array}{c}
\mathcal{T}_{K}(t, \rho) \int_{s}^{\rho} \mathcal{T}_{K}(\rho, \tau) \mathcal{K}(\tau) \widehat{\Phi}(\tau, s) u_{0} d \tau \\
0
\end{array}\right) \\
& +\left(\begin{array}{c}
\int_{\rho}^{t} \mathcal{T}_{K}(t, \tau) \mathcal{L}(\tau)\left(R_{\tau, \rho} u_{1}+S_{U}(\tau-\rho) R_{\rho, s} u_{0}\right) d \tau \\
R_{t, \rho} u_{1}+S_{U}(t-\rho) R_{\rho, s} u_{0}
\end{array}\right) \\
& +\left(\begin{array}{c}
\mathcal{T}_{K}(t, \rho) \int_{s}^{\rho} \mathcal{T}_{K}(\rho, \tau) \mathcal{L}(\tau) R_{\tau, s} u_{0} d \tau \\
0
\end{array}\right) \\
= & \Phi_{K, L}(t, \rho) u_{1}+\left(\begin{array}{c}
\mathcal{T}_{K}(t, \rho)\left[\widehat{\Phi}(\rho, s) u_{0}+\int_{s}^{\rho} \mathcal{T}_{K}(\rho, \tau) \mathcal{K}(\tau) \widehat{\Phi}(\tau, s) u_{0} d \tau\right] \\
0
\end{array}\right) \\
+ & \left(\begin{array}{c}
\left.\mathcal{T}_{K}(t, \rho) \int_{s}^{\rho} \mathcal{T}_{K}(\rho, \tau) \mathcal{L}(\tau) R_{\tau, s} u_{0} d \tau+\int_{\rho}^{t} \mathcal{T}_{K}(t, \tau) \mathcal{L}(\tau) S_{U}(\tau-\rho) R_{\rho, s} u_{0} d \tau\right) \\
S_{U}(t-\rho) R_{\rho, s} u_{0}
\end{array}\right) \\
= & \Phi_{K, L}(t, \rho) u_{1}+\mathcal{T}_{K, L}(t, \rho) \Phi_{K, L}(\rho, s) u_{0} .
\end{aligned}
$$

The set of the above used $u$ is dense in $L_{l o c}^{2}([s, \infty), U)$, so that the assertion follows by approximation.

In view of Proposition 3.5(c) the third summand in (4.10) gives the delayed input to the retarded problem solved by $\mathcal{T}_{K}$. In order to interpret the other two summands of $\Phi_{K, L}$, we introduce the operators

$$
\Phi_{K}(t, s) u:=\widehat{\Phi}(t, s) u+\int_{s}^{t} \mathcal{T}_{K}(t, \tau) \mathcal{K}(\tau) \widehat{\Phi}(\tau, s) u d \tau
$$

for $t \geq s \geq 0$ and $u \in L_{l o c}^{2}([s, \infty), U)$. The proof Proposition 4.4 with $L(t)=0$ also yields that $\left(\mathcal{T}_{K}, \Phi_{K}\right)$ is a time varying control system on $U$ and $\mathcal{X}_{0}$. It describes the effect of the given input $\Phi$ to the delay system solved by $\mathcal{T}_{K}$. To see this more clearly, we observe that there are bounded control operators $B_{n}(t) \in \mathcal{L}(U, X)$ such that

$$
\widehat{\Phi}(t, s) u=\lim _{n \rightarrow \infty} \int_{s}^{t} \mathcal{T}(t, \sigma)\left(\begin{array}{c}
B_{n}(\sigma) u(\sigma) \\
0
\end{array}\right) d \sigma=\lim _{n \rightarrow \infty} \int_{s}^{t}\left(\begin{array}{c}
T(t, \sigma) B_{n}(\sigma) u(\sigma) \\
T_{t, \sigma} B_{n}(\sigma) u(\sigma)
\end{array}\right) d \sigma
$$

for $u \in L_{l o c}^{2}([s, \infty), u)$ and $t \geq s \geq 0$, due to (2.3). This limit exists in $\mathcal{D}_{0}$ locally uniformly in $t$. Hence, (4.13), Propositions 2.1 and 2.2, Fubini's theorem, and (3.6) yield

$$
\begin{aligned}
\Phi_{K}(t, s) u= & \lim _{n \rightarrow \infty} \int_{s}^{t} \mathcal{T}(t, \sigma)\left(\begin{array}{c}
B_{n}(\sigma) u(\sigma) \\
0
\end{array}\right) d \sigma \\
& +\lim _{n \rightarrow \infty} \lim _{k \rightarrow \infty} \int_{s}^{t} \mathcal{T}_{K}(t, \tau) \mathcal{K}_{\frac{1}{k}}(\tau) \int_{s}^{\tau} \mathcal{T}(\tau, \sigma)\left(\begin{array}{c}
B_{n}(\sigma) u(\sigma) \\
0
\end{array}\right) d \sigma d \tau \\
= & \lim _{n \rightarrow \infty} \int_{s}^{t}\left[\mathcal{T}(t, \sigma)+\int_{\sigma}^{t} \mathcal{T}_{K}(t, \tau) \widetilde{\mathcal{K}}(\tau) \mathcal{T}(\tau, \sigma) d \tau\right]\left(\begin{array}{c}
B_{n}(\sigma) u(\sigma) \\
0
\end{array}\right) d \sigma \\
= & \lim _{n \rightarrow \infty} \int_{s}^{t} \mathcal{T}_{K}(t, \sigma)\left(\begin{array}{c}
B_{n}(\sigma) u(\sigma) \\
0
\end{array}\right) d \sigma
\end{aligned}
$$

for $t \geq s \geq 0$ and $u \in L_{l o c}^{2}([s, \infty), U)$. Consequently, also $\Phi_{K}$ can represented by the 'approximative control operators' $B_{n}(t)$. 
Let $(x, f, g) \in \mathcal{D}$ and $u \in C([s, \infty), U)$ with $u(s)=0$. Let $\Pi: \mathcal{X} \rightarrow \mathcal{X}_{0}$ be the canonical projection. Then the above observations combined with (4.2) and (4.10) yield

$$
\begin{aligned}
\Pi\left[\mathcal{T}_{K, L}(t, s)(x, f, g)+\Phi_{K, L}(t, s) u\right]= & \mathcal{T}_{K}(t, s)\left(\begin{array}{l}
x \\
f
\end{array}\right)+\lim _{n \rightarrow \infty} \int_{s}^{t} \mathcal{T}_{K}(t, \tau)\left(\begin{array}{c}
B_{n}(\sigma) u(\sigma) \\
0
\end{array}\right) d \sigma \\
& +\int_{s}^{t} \mathcal{T}_{K}(t, \tau) \mathcal{L}(\tau)\left(R_{\tau, s} u+S_{U}(\tau-s) g\right) d \tau
\end{aligned}
$$

This formula can be extended to data $\left(\begin{array}{l}x \\ f\end{array}\right) \in \mathcal{D}_{0}, g \in C([-1,0], U)$, and $u \in C([s, \infty), U)$ with $u(s)=g(0)$ using Lemma 4.3. Replacing $\mathcal{L}(\tau)$ by $\overline{\mathcal{L}}(\tau)$ the identity (4.15) is also valid for $(x, f, g) \in \mathcal{X}$ and $u \in L^{2}([s, \infty), U)$. In view of Proposition 3.5(c), formula (4.15) gives (in an approximative sense) the mild solutions of (nLDS) without observations.

Finally, we suppose that $(T, \Phi, \Psi, \mathbb{F})$ is a time varying regular system. We introduce the (canonical) input-output operators for $\Psi_{K, L}$ and $\Phi_{K, L}$ by setting

$$
\mathbb{F}_{K, L}(s) u=\mathbf{C}(\cdot) \Phi_{K, L}(\cdot, s) u
$$

for $u \in L_{l o c}^{2}([s, \infty), U)$ and $s \geq 0$, where $\mathbf{C}(t)$ was defined in Proposition 4.2. Theorem 3.11 of Schnaubelt $(2002 \mathrm{~b})$ shows that $\mathbb{F}(s)=\widetilde{C}(\cdot) \Phi(\cdot, s) u$ is a well-defined operator. Moreover, Lemma 4.1, Proposition 2.2, and Lemma 4.3 imply that one can apply $\mathbf{C}(t)$ to the second and third summand in the definition of $\Phi_{K, L}(t, s) u$. Thus, the operators $\mathbb{F}_{K, L}(s): L_{l o c}^{2}([s, \infty), U) \rightarrow L_{l o c}^{2}([s, \infty), Y)$ are well-defined. In view of (4.15) and (4.16), $\mathbb{F}_{K, L}(s)$ are the input-output operators of (nDLS).

Theorem 4.5. Assume that $\Sigma=(T, \Psi, \Phi, \mathbb{F})$ is a time varying regular system on the spaces $U, X$, and $Y$ with representing operators $\widetilde{C}(t)$ and that the kernels $k$ and $l$ satisfy assumption $(H)$ for $X=Z$ and $U=Z$, respectively. Then $\Sigma_{K, L}=$ $\left(\mathcal{T}_{K, L}, \Psi_{K, L}, \Phi_{K, L}, \mathbb{F}_{K, L}\right)$ defined in (4.2), (4.6), (4.11), and (4.16) is a regular system on $U, \mathcal{X}$, and $Y$. It is absolutely regular if and only if $\mathbb{F}$ is absolutely regular. Finally, $\Delta(\cdot) \in L^{\infty}\left(\mathbb{R}_{+}, \mathcal{L}_{s}(Y, U)\right)$ is an admissible feedback for $\mathbb{F}_{K, L}$ if and only if it is an admissible feedback for $\mathbb{F}$.

Proof. The operators $\mathbb{F}_{K, L}(s)$ satisfy the estimate (2.9) for $s \geq 0$ due to Theorem 3.11 of Schnaubelt (2002b), Lemma 4.1, Proposition 2.2, and Lemma 4.3. Next, for $u$ given as in (4.7), we obtain

$$
\begin{aligned}
\mathbb{F}_{K, L}(s) u & =\mathbf{C}(\cdot) \Phi_{K, L}(\cdot, t) u_{1}+\mathbf{C}(\cdot) \mathcal{T}_{K, L}(\cdot, t) \Phi_{K, L}(t, s) u_{0} \\
& =\mathbb{F}_{K, L}(t) u_{1}+\Psi_{K, L}(t) \Phi_{K, L}(t, s) u_{0},
\end{aligned}
$$

due to Propositions 4.2 and 4.4. Thus $\left(\mathcal{T}_{K, L}, \Phi_{K, L}, \Psi_{K, L}, \mathbb{F}_{K, L}\right)$ is a well-posed time varying system. To check the regularity of the system, we set $u_{z}(\sigma)=z$ for $z \in U$ and $\sigma \geq 0$. Let $s \geq 0$ and $0 \leq t \leq t_{1}$. At first, we note that

$$
\frac{1}{t} \int_{s}^{s+t} \mathbf{C}(\sigma)\left(\begin{array}{c}
\widehat{\Phi}(\sigma, s) u_{z} \\
0
\end{array}\right) d \sigma=\frac{1}{t} \int_{19}^{s+t} \widetilde{C}(\sigma) \Phi(\sigma, s) u_{z} d \sigma \longrightarrow 0
$$


as $t \searrow 0$ by the regularity of $\mathbb{F}(s)$. Lemma 4.1 and Proposition 2.2 allow to estimate

$$
\begin{aligned}
\frac{1}{t} \int_{s}^{s+t}\left\|\mathcal{C}(\sigma)\left(\mathbb{K}_{s}^{\mathcal{T}_{K}} \mathcal{K}(\cdot) \hat{\Phi}(\cdot, s) u_{z}\right)(\sigma)\right\|^{2} d \sigma & \leq c \int_{s}^{s+t}\left\|\mathcal{K}(\sigma) \hat{\Phi}(\sigma, s) u_{z}\right\|^{2} d \sigma \\
& \leq c t \sup _{s \leq \sigma \leq s+t}\left\|\Phi(\sigma, s) u_{z}\right\|^{2} \leq c t^{2}\|z\|^{2} .
\end{aligned}
$$

Here and below the constants $c>0$ only depend on $\Sigma$, the kernels $k$ and $l$, and (possibly) on $t_{1}>0$. Take functions $\alpha_{n} \in C([s, \infty))$ such that $0 \leq \alpha_{n} \leq 1, \alpha_{n}(s)=0$, and $\alpha_{n}(t)=1$ for $t \geq s+\frac{1}{n}, n \in \mathbb{N}$, and set $u_{n}=\alpha_{n} u_{z}$. Observe that $u_{n} \rightarrow u_{z}$ in $L_{l o c}^{2}([s, \infty), U)$ so that

$$
\begin{aligned}
\int_{s}^{s+t}\left\|\bar{L}(\sigma) R_{\sigma, s} u_{z}\right\|^{2} d \sigma & =\lim _{n \rightarrow \infty} \int_{s}^{s+t}\left\|L(\sigma) R_{\sigma, s} u_{n}\right\|^{2} d \sigma \\
& \leq c_{l}^{2} \limsup _{n \rightarrow \infty} \int_{s}^{s+t}\left\|R_{\sigma, s} u_{n}\right\|_{\infty}^{2} d \sigma \leq c_{l}^{2} t\|z\|^{2} .
\end{aligned}
$$

This estimate and the same arguments as above imply that

$$
\frac{1}{t} \int_{s}^{s+t}\left\|\mathcal{C}(\sigma)\left(\mathbb{K}_{s}^{\mathcal{T}_{K}}\left(\begin{array}{c}
\bar{L}(\cdot) R_{\bullet}, s u_{z} \\
0
\end{array}\right)\right)(\sigma)\right\|^{2} d \sigma \leq c t\|z\|^{2} .
$$

As a result, $\left(\mathcal{T}_{K, L}, \Phi_{K, L}, \Psi_{K, L}, \mathbb{F}_{K, L}\right)$ is regular. Moreover, its absolute regularity is equivalent to the absolute regularity of $(T, \Phi, \Psi, \mathbb{F})$ due to estimates (4.17) and (4.18). We finally deduce from (4.11), Proposition 2.2, and Lemma 4.3 that

$$
\begin{aligned}
\left\|\left(\mathbb{F}_{K, L}\left(s+t_{0}, s\right)-\mathbb{F}\left(s+t_{0}, s\right)\right) \Delta(\cdot) v\right\|_{L^{2}\left(\left[s, s+t_{0}\right], Y\right)} \\
\quad \leq c t_{0}^{\frac{1}{2}}\left(\|\mathcal{K}(\cdot) \widehat{\Phi}(\cdot, s) \Delta(\cdot) v\|_{L^{2}\left(\left[s, s+t_{0}\right], X\right)}+\left\|\overline{\mathcal{L}}(\cdot) R_{\bullet, s} \Delta(\cdot) v\right\|_{L^{2}\left(\left[s, s+t_{0}\right], X\right)}\right) \\
\quad \leq c t_{0}^{\frac{1}{2}}\|v\|_{L^{2}\left(\left[s, s+t_{0}\right], Y\right)}
\end{aligned}
$$

for $v \in L_{l o c}^{2}([s, \infty), Y), t_{0}>0$, and $s \geq 0$. Thus, the above estimate yields the assertion concerning the admissibility of $\Delta(\cdot)$ if we take a sufficiently small $t_{0}>0$.

\section{THE FEEDBACK PROBLEM}

We now assume that the hypotheses of Theorem 4.5 hold for an absolutely regular system $\Sigma$ with an admissible feedback $\Delta(\cdot)$. We want to solve the feedback problem (formally) given by (nLDS) with $u(t)=\Delta(t) \tilde{C}(t) w(t)$. As in (3.2) and (4.15) we are looking for a mild solution of the feedback problem, namely for a function $w \in C([s, \infty), X)$ such that $w \in D_{s}(\tilde{C}(\cdot))$ and

$$
\begin{aligned}
w(t)= & T(t, s) x+\int_{s}^{t} T(t, \tau) K(\tau) w_{\tau} d \tau+\lim _{n \rightarrow \infty} \int_{s}^{t} T(t, \tau) B_{n}(\tau) \Delta(\tau) \widetilde{C}(\tau) w(\tau) d \tau \\
& +\int_{s}^{t} T(t, \tau) \bar{L}(\tau)\left(R_{\tau, s}[\Delta(\cdot) \tilde{C}(\cdot) w(\cdot)]+S_{U}(\tau-s) g\right) d \tau, \quad t \geq s \geq 0, \quad(5.1) \\
w(s+\theta) & =f(\theta), \quad-1 \leq \theta \leq 0,
\end{aligned}
$$


where $\left(\begin{array}{l}x \\ f\end{array}\right) \in \mathcal{D}_{0}$ with $\tilde{C}(\cdot) f \in L^{2}([-1,0], Y)$ are given, the operators $B_{n}(t)$ were defined before (2.3), and we set

$$
g(\theta):=u(s+\theta)=\Delta(s+\theta) \tilde{C}(s+\theta) w(s+\theta)=\Delta(s+\theta) \tilde{C}(s+\theta) f(s+\theta)
$$

for $\theta \in[-1,0]$. The function $g$ is the prehistory of the 'input' $u(t)=\Delta(t) \tilde{C}(t) w(t)$ given by the data of the closed loop system. Observe that we need the extensions $\bar{L}(t)$ (defined after Lemma 4.3) since it is not clear whether $g$ is continuous. Due to (2.3), the summand involving $B_{n}(t)$ can be replaced by

$$
\lim _{n \rightarrow \infty} \int_{s}^{t} T(t, \tau) B_{n}(\tau) \Delta(\tau) \tilde{C}(\tau) w(\tau) d \tau=\Phi(t, s) \Delta(\cdot) \tilde{C}(\cdot) w(\cdot) .
$$

In order to state our final result, we further set

$$
\left(\mathcal{B}_{n} u\right)(t):=n \Phi\left(t, t-\frac{1}{n}\right) u
$$

for $t \geq 0, n \in \mathbb{N}$, and $u \in L_{l o c}^{2}\left(\mathbb{R}_{+}, U\right)$. This variant of the operators $B_{n}(t)$ is needed to approximate the input-output operators, see Proposition 3.12 of Schnaubelt (2002b).

Theorem 5.1. Assume that $\Sigma=(T, \Psi, \Phi, \mathbb{F})$ is a time varying absolutely regular system on the spaces $U, X$, and $Y$ with representing operators $\widetilde{C}(t)$ and an admissible feedback $\Delta(\cdot) \in L^{\infty}\left(\mathbb{R}_{+}, \mathcal{L}_{s}(Y, U)\right)$. Further suppose that the kernels $k$ and $l$ satisfy assumption (H) for $X=Z$ and $U=Z$, respectively. Let $\Sigma_{K, L}=\left(\mathcal{T}_{K, L}, \Psi_{K, L}, \Phi_{K, L}, \mathbb{F}_{K, L}\right)$ be defined in (4.2), (4.6), (4.11), and (4.16) on $U, \mathcal{X}$, and $Y$. Then there is a unique absolutely regular system $\Sigma_{K, L}^{\Delta}=\left(\mathcal{T}_{K, L}^{\Delta}, \Psi_{K, L}^{\Delta}, \Phi_{K, L}^{\Delta}, \mathbb{F}_{K, L}^{\Delta}\right)$ on $U, \mathcal{X}$, and $Y$ such that the observation operators $\mathbf{C}(\cdot)$ of $\Sigma_{K, L}$ are admissible for $\mathcal{T}_{K, L}^{\Delta}$ and

$$
\begin{aligned}
\Sigma_{K, L}^{\Delta}(t, s) & =\Sigma_{K, L}(t, s)+\Sigma_{K, L}(t, s)\left(\begin{array}{cc}
0 & 0 \\
0 & \Delta(\cdot)
\end{array}\right) \Sigma_{K, L}^{\Delta}(t, s) \\
& =\Sigma_{K, L}(t, s)+\Sigma_{K, L}^{\Delta}(t, s)\left(\begin{array}{cc}
0 & 0 \\
0 & \Delta(\cdot)
\end{array}\right) \Sigma_{K, L}(t, s)
\end{aligned}
$$

for $t \geq s \geq 0$ (where we use an analogous notation as in (2.10)). In particular, the left upper components of (5.4) and (5.5) yield

$$
\mathcal{T}_{K, L}^{\Delta}(t, s)-\mathcal{T}_{K, L}(t, s)=\Phi_{K, L}(t, s) \Delta(\cdot) \Psi_{K, L}^{\Delta}(s)=\Phi_{K, L}^{\Delta}(t, s) \Delta(\cdot) \Psi_{K, L}(s) .
$$

Moreover, we have

$$
\begin{aligned}
\mathcal{T}_{K, L}^{\Delta}(t, s) & =\mathcal{T}_{K, L}(t, s)+\Phi_{K, L}(t, s) \Delta(\cdot)\left(I-\mathbb{F}_{K, L}\left(s+t_{0}, s\right) \Delta(\cdot)\right)^{-1} \Psi_{K, L}(s) \\
\Psi_{K, L}^{\Delta}(s) & =\mathbf{C}(\cdot) \mathcal{T}_{K, L}^{\Delta}(\cdot, s) \\
\Pi \Phi_{K, L}^{\Delta}(t, s) u & =\lim _{n \rightarrow \infty} \Pi \int_{s}^{t} \mathcal{T}_{K, L}^{\Delta}(t, \tau)\left(\begin{array}{c}
\left(\mathcal{B}_{n} u\right)(\tau)+\bar{L}(\tau) R_{\tau, s} u \\
0 \\
0
\end{array}\right) d \tau \\
\mathbb{F}_{K, L}^{\Delta}(s) u & =\mathbf{C}(\cdot) \Phi_{K, L}^{\Delta}(\cdot, s)=\lim _{n \rightarrow \infty} \mathbf{C}(\cdot) \int_{s}^{\bullet} \mathcal{T}_{K, L}^{\Delta}(\cdot, \tau)\left(\begin{array}{c}
\left(\mathcal{B}_{n} u\right)(\tau)+\bar{L}(\tau) R_{\tau, s} u \\
0 \\
0
\end{array}\right) d \tau
\end{aligned}
$$

for $0 \leq s \leq t \leq s+t_{0}, t_{0} \geq 0$, and $u \in L_{\text {loc }}^{2}\left(\mathbb{R}_{+}, U\right)$, where $\Pi: \mathcal{X} \rightarrow \mathcal{X}_{0}$ is the canonical projection, the first limit is taken in $X$ locally uniformly in $t \geq s$, and the second one is 
taken in $L_{l o c}^{2}([s, \infty), Y)$. Finally, the unique mild solution $w$ of the feedback problem (5.1) is given by

$$
\left(\begin{array}{c}
w(t) \\
w_{t}
\end{array}\right)=\Pi \mathcal{T}_{K, L}^{\Delta}(t, s)\left(\begin{array}{c}
x \\
f \\
g
\end{array}\right), \quad t \geq s \geq 0,
$$

for $\left(\begin{array}{l}x \\ f\end{array}\right) \in \mathcal{D}_{0}$ with $f(t) \in D(\tilde{C}(t))$ for a.e. $t \in[-1,0]$ and $\tilde{C}(\cdot) f \in L^{2}([-1,0], Y)$, where $g$ is given by (5.2).

Proof. By virtue of Theorem 4.5 most of the assertions follow from Theorem 4.4 and Proposition 5.1 in Schnaubelt (2002b). It remains to verify the last assertion and the approximation formulas for $\Phi_{K, L}^{\Delta}$ and $\mathbb{F}_{K, L}^{\Delta}$ in (5.9) and (5.10). Let $u \in L_{l o c}^{2}\left(\mathbb{R}_{+}, U\right)$ and $t \geq s \geq 0$. Propositions 3.5 and 3.12 of Schnaubelt (2002b) show that

$$
\mathbb{K}_{s}^{T} \mathcal{B}_{n} u(t) \rightarrow \Phi(t, s) u \quad \text { and } \quad \tilde{C}(\cdot) \mathbb{K}_{s}^{T} \mathcal{B}_{n} u \rightarrow \mathbb{F}(s) u
$$

where the first limit is taken in $X$ locally uniformly in $t \geq s$ and the second one is taken in $L_{l o c}^{2}([s, \infty), Y)$. As a consequence, one can replace in (4.14) and (4.15) the term $B_{n}(\sigma) u(\sigma)$ by $\left(\mathcal{B}_{n} u\right)(\sigma)$. Combining these facts with (4.11), we see that

$$
\Phi_{K, L}(t, s) u=\lim _{n \rightarrow \infty}\left(\int_{s}^{t} \mathcal{T}_{K}(t, \tau)\left[\left(\begin{array}{c}
\left(\mathcal{B}_{n} u\right)(\tau) \\
0
\end{array}\right)+\left(\begin{array}{c}
\bar{L}(\tau) R_{\tau, s} u \\
0
\end{array}\right)\right] d \tau, R_{t, s} u\right)^{T} .
$$

Because of (5.11) and Lemma 4.1, the equation (4.14) (with $B_{n}(\cdot) u$ replaced by $\mathcal{B}_{n} u$ ) remains valid if one applies $\mathcal{C}(t)$ and takes the limit in $L_{l o c}^{2}([s, \infty), Y)$. Hence,

$$
\mathcal{C}(\cdot) \Phi_{K}(\cdot, s) u=\lim _{n \rightarrow \infty} \mathcal{C}(\cdot) \mathbb{K}_{s}^{\mathcal{T}_{K}}\left(\begin{array}{c}
\mathcal{B}_{n} u \\
0
\end{array}\right) .
$$

From this equation and (4.16), $\mathbf{C}(t)=(\mathcal{C}(t), 0)^{T},(5.12)$, (4.2), we further deduce that

$$
\mathbb{F}_{K, L}(s) u=\mathbf{C}(\cdot) \Phi_{K, L}(\cdot, s) u=\lim _{n \rightarrow \infty} \mathbf{C}(\cdot) \mathbb{K}_{s}^{\mathcal{T}_{K, L}}\left(\begin{array}{c}
\mathcal{B}_{n} u+\bar{L}(\cdot) R \bullet, s u \\
0 \\
0
\end{array}\right) .
$$

The formulas (5.12) and (5.13) together with the upper right component of (5.5) lead to

$$
\begin{aligned}
\Pi \Phi_{K, L}^{\Delta}(t, s) u & =\lim _{n \rightarrow \infty} \mathbb{K}_{s}^{\mathcal{T}_{K}}\left(\begin{array}{c}
\mathcal{B}_{n} u+\bar{L}(\cdot) R_{\bullet}, s \\
0
\end{array}\right)+\lim _{n \rightarrow \infty} \Pi \Phi_{K, L}^{\Delta}(t, s) \Delta(\cdot) \mathbf{C}(\cdot) \mathbb{K}_{s}^{\mathcal{T}_{K, L}}\left(\begin{array}{c}
\mathcal{B}_{n} u+\bar{L}(\cdot) R \bullet, s u \\
0 \\
0
\end{array}\right) \\
& =\lim _{n \rightarrow \infty} \Pi\left[\mathbb{K}_{s}^{\mathcal{T}_{K, L}}+\Phi_{K, L}^{\Delta}(t, s) \Delta(\cdot) \mathbf{C}(\cdot) \mathbb{K}_{s}^{\mathcal{T}_{K, L}}\right]\left(\begin{array}{c}
\mathcal{B}_{n} u+\bar{L}(\cdot) R \bullet, s u \\
0 \\
0
\end{array}\right),
\end{aligned}
$$

where the limits are locally uniform in $t$. On the other hand, $-\Delta(\cdot)$ is an admissible feedback for $\Sigma_{K, L}^{\Delta}$ and $\left(\Sigma_{K, L}^{\Delta}\right)^{-\Delta}=\Sigma_{K, L}$ by Proposition 5.4 of Schnaubelt (2002b). Applying equation (4.12) of Schnaubelt (2002b) to this setting, we obtain

$$
\mathbb{K}_{s}^{\mathcal{T}_{K, L}} h=\mathbb{K}_{s}^{\mathcal{T}_{K, L}^{\Delta}} h-\Phi_{K, L}^{\Delta}(\cdot, s) \Delta(\cdot) \mathbf{C}(\cdot) \mathbb{K}_{s}^{\mathcal{T}_{K, L}} h
$$

for $h \in L_{l o c}^{2}([s, \infty), \mathcal{X})$. (We note that (4.12) in Schnaubelt (2002b) is formulated for $h$ contained in a dense subspace, but Proposition 2.2 allows to pass to general $h \in L_{l o c}^{2}([s, \infty), \mathcal{X})$ by approximation.) Then equation $(5.9)$ is a consequence of $(5.14)$ and (5.15). Moreover, (5.15), Proposition 2.2, and the first part of (5.10) yield

$$
\mathbf{C}(\cdot) \mathbb{K}_{s}^{\mathcal{T}_{K, L}}=\mathbf{C}(\cdot) \mathbb{K}_{s}^{\mathcal{T}_{K, L}^{\Delta}}-\mathbb{F}_{K, L}^{\Delta}(s) \Delta(\cdot) \mathbf{C}(\cdot) \mathbb{K}_{s}^{\mathcal{T}_{K, L}}
$$

Now the second equation in (5.10) can be shown as in (5.14) using (5.13). 
To construct a solution of $(5.1)$, we take $s \geq 0$ and $\left(\begin{array}{l}x \\ f\end{array}\right) \in \mathcal{D}_{0}$ with $\tilde{C}(\cdot) f \in$ $L^{2}([-1,0], Y)$. Define $g$ by $(5.2), w(t)=f(t-s)$ for $s-1 \leq t \leq s$, and

$$
\left(\begin{array}{c}
w(t) \\
W(t)
\end{array}\right)=\Pi \mathcal{T}_{K, L}^{\Delta}(t, s)\left(\begin{array}{l}
x \\
f \\
g
\end{array}\right) \quad \text { for } t \geq s .
$$

Then $w \in C([s-1, \infty), X)$ and $w \in D_{s}(\tilde{C}(\cdot))$. Formulas (5.6), (4.15), and (5.8) yield

$$
\begin{array}{r}
\left(\begin{array}{c}
w(t) \\
W(t)
\end{array}\right)=\mathcal{T}_{K}(t, s)\left(\begin{array}{l}
x \\
f
\end{array}\right)+\lim _{n \rightarrow \infty} \int_{s}^{t} \mathcal{T}_{K}(t, \sigma)\left(\begin{array}{c}
B_{n}(\sigma) \Delta(\sigma) \tilde{C}(\sigma) w(\sigma) \\
0
\end{array}\right) d \sigma \\
+\int_{s}^{t} \mathcal{T}_{K}(t, \tau) \overline{\mathcal{L}}(\tau)\left[R_{\tau, s}(\Delta(\cdot) \tilde{C}(\cdot) w(\cdot))+S_{U}(\tau-s) g\right] d \tau
\end{array}
$$

We denote by $\left(w^{n}(t), W^{n}(t)\right)$ the right hand side of this equation without the limit and set $w^{n}(t)=f(t-s)$ for $s-1 \leq t \leq s$. Then Proposition 3.5(c) shows that $W^{n}(t)=w_{t}^{n}$ and that $w^{n}$ satisfies

$$
\begin{aligned}
w^{n}(t)=T( & t, s) x+\int_{s}^{t} T(t, \tau) K(\tau) w_{\tau}^{n} d \tau+\int_{s}^{t} T(t, \tau) B_{n}(\tau) \Delta(\tau) \tilde{C}(\tau) w(\tau) d \tau \\
& +\int_{s}^{t} T(t, \tau) \bar{L}(\tau)\left(R_{\tau, s}[\Delta(\cdot) \tilde{C}(\cdot) w(\cdot)]+S_{U}(\tau-s) g\right) d \tau, \quad t \geq s \geq 0
\end{aligned}
$$

Thus, $w$ solves (5.1) since $w^{n}(t)$ tends to $w(t)$ locally uniformly in $t \geq s-1$ as $n \rightarrow \infty$.

To prove uniqueness of solutions to (5.1), we suppose that $w$ solves (5.1) with $x=0$ and $f=0$. Hence, $g=0, w=0$ on $[s-1, s]$, and using (5.3) we get

$$
w(t)=\int_{s}^{t} T(t, \tau)\left\{K(\tau) w_{\tau}+\bar{L}(\tau)[\Delta(\cdot) \tilde{C}(\cdot) w(\cdot)]_{\tau}\right\} d \tau+\Phi(t, s) \Delta(\cdot) \tilde{C}(\cdot) w
$$

for $t \geq s$. We can apply $\tilde{C}(\cdot)$ to $(5.16)$. The admissibility of $\Delta(\cdot)$ then implies

$$
\tilde{C}(\cdot) w=\left[I-\mathbb{F}\left(s+t_{0}, s\right) \Delta(\cdot)\right]^{-1} \tilde{C}(\cdot) \mathbb{K}_{s}^{T}\left\{K(\cdot) w_{\bullet}+\bar{L}(\cdot)[\Delta(\cdot) \tilde{C}(\cdot) w(\cdot)]_{\bullet}\right\},
$$

where we may take any $t_{0}>0$ due to Lemma 4.2 of Schnaubelt (2002b). Proposition 2.2 and Lemma 4.3 allow us to estimate

$$
\begin{aligned}
\|\tilde{C}(\cdot) w\|_{L^{2}\left(\left[s, s+t_{0}\right], Y\right)} & \leq c t_{0}^{\frac{1}{2}}\left(\left\|K(\cdot) w_{\bullet}\right\|_{L^{2}\left(\left[s, s+t_{0}\right], X\right)}+\|\bar{L}(\cdot)[\Delta(\cdot) \tilde{C}(\cdot) w] \bullet\|_{L^{2}\left(\left[s, s+t_{0}\right], X\right)}\right) \\
& \leq c t_{0}\|w\|_{L^{\infty}\left(\left[s, s+t_{0}\right], X\right)}+c t_{0}^{\frac{1}{2}}\|\tilde{C}(\cdot) w\|_{L^{2}\left(\left[s, s+t_{0}\right], Y\right)} .
\end{aligned}
$$

Here and below the constants $c>0$ are independent of $s \geq 0$ and $0<t_{0} \leq t_{1}$, where $t_{1}$ is fixed. Taking a small $t_{0}>0$, we thus obtain

$$
\|\tilde{C}(\cdot) w\|_{L^{2}\left(\left[s, s+t_{0}\right], Y\right)} \leq c t_{0}\|w\|_{L^{\infty}\left(\left[s, s+t_{0}\right], X\right)} .
$$

Identity (5.16) combined with Lemma 4.3 and (5.17) yield

$$
\max _{s \leq t \leq s+t_{0}}\|w(t)\| \leq c t_{0}\|w\|_{L^{\infty}\left(\left[s, s+t_{0}\right], X\right)} .
$$

If we decrease $t_{0}>0$ once more, we see that $w(t)=0$ for $s \leq t \leq s+t_{0}$. This procedure can be iterated with the same $t_{0}>0$, so that $w=0$. 
In order to illustrate the above results, we consider a parabolic problem with delays and boundary control and observation, where we concentrate on a simplified case.

Example 5.2. We consider the controlled partial differential equation

$$
\begin{aligned}
& \partial_{t} w(t, x)=\operatorname{div}(a(t, x) \nabla w(t, x))+w(t-r(t), x)+u_{1}(t-\rho(t), x), \quad x \in \Omega, \quad t>0, \\
& (a(t, x) \nabla w(t, x) \mid \nu(x))=u_{2}(t, x), \quad x \in \partial \Omega, \quad t>0, \\
& y(t, x)=c(t, x) w(t, x), \quad x \in \partial \Omega, \quad t \geq 0, \\
& w(t, x)=f(t, x), \quad u_{1}(t, x)=g_{1}(t, x), \quad u_{2}\left(t, x^{\prime}\right)=g_{2}\left(t, x^{\prime}\right), \quad-1 \leq t \leq 0, x \in \Omega, x^{\prime} \in \partial \Omega .
\end{aligned}
$$

Here $\Omega$ is a bounded open subset of $\mathbb{R}^{n}$ with a $C^{2}$ boundary $\partial \Omega$ and outer unit normal $\nu(x), a(t, x)=\left[a_{i j}(t, x)\right] \in \mathbb{R}^{d \times d}$ with $a_{i j}=a_{j i} \in C_{b}^{1}\left(\mathbb{R}_{+}, C^{1}(\bar{\Omega})\right)$ with $a(t, x) \geq \eta I$ for some $\eta>0$ and all $(t, x) \in \mathbb{R}_{+} \times \bar{\Omega}$, the functions $r, \rho \in C^{1}([-1, \infty))$ take values in $[-1,0]$ and $r^{\prime}, \rho^{\prime} \leq 1-\delta$ for some $\delta>0, c \in C_{b}\left(\mathbb{R}_{+} \times \partial \Omega\right), f, g_{1} \in C([-1,0] \times \bar{\Omega})$, and $g_{2} \in C([-1,0] \times \partial \Omega)$. We set $X=L^{2}(\Omega), Y=L^{2}(\partial \Omega), U=L^{2}(\Omega) \times L^{2}(\partial \Omega), K(t) w_{t}=$ $w(t-r(t))$ for $w:[-1, \infty) \rightarrow X$, and $L(t) u_{t}=u_{1}(t-\rho(t))$ for $u=\left(u_{1}, u_{2}\right):[-1, \infty) \rightarrow U$. These delay operators satisfy hypothesis $(H)$ due to Example 3.3. We further introduce

$$
\begin{aligned}
A_{0}(t) \varphi & :=\operatorname{div}(a(t, \cdot) \nabla \varphi), \\
D\left(A_{0}(t)\right) & :=\left\{\varphi \in W^{2,2}(\Omega):(a(t, x) \nabla \varphi(x) \mid \nu(x))=0, x \in \partial \Omega\right\} .
\end{aligned}
$$

It is known that the operators $A_{0}(t), t \geq 0$, generate an evolution family $T(t, s), t \geq s \geq 0$, on $X$ such that

$$
\left\|\left(I-A_{0}(t)\right)^{\alpha} T(t, s)\left(I-A_{0}(s)\right)^{\beta} x\right\| \leq c e^{\omega(t-s)} \max \left\{1,(t-s)^{-\alpha-\beta}\right\}\|x\|
$$

for $t>s \geq 0, \alpha, \beta \in[0,1], x \in D\left(\left(I-A_{0}(t)\right)^{\beta}\right)$, and some constants $c, \omega \geq 0$. We further let $X_{-\gamma}^{t}$ be the completion of $X$ with respect to the norm $\left\|\left(I-A_{0}(t)\right)^{-\gamma} \varphi\right\|$ for $\gamma \in[0,1]$. We note that $X_{-1}^{t}$ is isomorphic to the dual space of the domain $D\left(A_{0}(t)\right)$ endowed with the graph norm of $A_{0}(t)$. By (5.19) we can extend $T(t, s)$ to a bounded operator $T(t, s): X_{-\beta}^{s} \rightarrow D\left(\left(I-A_{0}(t)\right)^{\alpha}\right)$ for $t>s$ and $\alpha, \beta \in[0,1]$. Moreover, one can extend $A_{0}(t)$ to a bounded operator $A_{-1}(t): X \rightarrow X_{-1}^{t}$, see Section II.5 in Engel and Nagel (2000). We further denote by $\varphi=: N(t) \psi \in W^{3 / 2,2}(\Omega)$ the solution of the elliptic boundary value problem

$$
\operatorname{div}(a(t, \cdot) \nabla \varphi)=0 \quad \text { on } \Omega, \quad(a(t) \nabla \varphi \mid \nu)=\psi \quad \text { on } \partial \Omega,
$$

where $\psi \in L^{2}(\partial \Omega)$. It is known that $N(t)$ maps $L^{2}(\partial \Omega)$ continuously into $D\left(\left(I-A_{0}(t)\right)^{\beta}\right)$ for $0<\beta<3 / 4$ and $t \geq 0$. Finally, the map

$$
(t, s) \mapsto\left(I-A_{0}(t)\right)^{\alpha} T(t, s)\left(I-A_{-1}(s)\right) N(s) \in \mathcal{L}\left(L^{2}(\partial \Omega), X\right)
$$

is continuous for $t>s$ and bounded by $c(\beta) e^{\omega(t-s)}(t-s)^{\beta-\alpha-1}$, where $\alpha \geq 0$ and $0<$ $\beta<3 / 4$, due to (5.19). See e.g. Section 9 of Acquistapace and Terreni (1999) and also Lasiecka and Triggiani (2000) for these facts. We can now define the input map

$$
\Phi(t, s) u=\int_{s}^{t} T(t, \tau)\left(I-A_{-1}(\tau)\right) N(\tau) u_{1}(\tau) d \tau
$$


for $u \in L_{l o c}^{2}\left(\mathbb{R}_{+}, U\right)$. As in Proposition 2.8 of Salamon (1987) one can show that a classical solution of (5.18) without delays, observation, and $f=g_{1}=g_{2}=0$ is given by $w=$ $\Phi(\cdot, 0) u_{2}$. The output map $\Psi$ is given by $\Psi(s) \varphi=C(\cdot) T(\cdot, s) \varphi$ for the observation operator $C(t) \varphi=c(t, \cdot) \operatorname{tr} \varphi$, where tr is the trace operator. Note that $C(t): D\left(\left(I-A_{0}(t)\right)^{\alpha}\right) \rightarrow Y$ is uniformly bounded, where $\alpha>1 / 4$. We finally define $\mathbb{F}(s)=C(\cdot) \Phi(\cdot, s)$. Using $(5.19)$ and taking $\alpha \in(1 / 4,1 / 2)$ and $\beta \in(1 / 2,3 / 4)$, it is then easy to verify that $\Sigma=(T, \Phi, \Psi, \mathbb{F})$ is an absolutely regular system. Since

$$
\left\|\mathbb{F}\left(s+t_{0}, s\right) u\right\|_{L^{2}\left(\left[s, s+t_{0}\right], Y\right)} \leq c t_{0}^{\beta-\alpha}\|u\|_{L^{2}\left(\left[s, s+t_{0}\right], Y\right)},
$$

every uniformly bounded, strongly measurable family $\Delta(t): Y \rightarrow U$ is an admissible feedback for $\Sigma$.

\section{REFERENCES}

Acquistapace, P., and Terreni, B. (1999) Classical solutions of nonautonomous Riccati equations arising in parabolic boundary control problems, Appl. Math. \& Optim. 39, 361-409.

Bátkai, A., and Piazzera, S. (2005) Semigroups for Delay Equations in $L^{p}$-Phase Spaces, A K Peters, Wellesley (Massachusetts).

Bensoussan, A., Da Prato, G., Delfour, M.C., and Mitter, S.K. (1992) Representation and Control of Infinite Dimensional Systems, Volumes I and II, Birkhäuser, Basel.

Chicone, C., and Latushkin, Y. (1999) Evolution Semigroups in Dynamical Systems and Differential Equations, Amer. Math. Soc., Providence (RI).

Da Prato, G., and Lunardi, A. (1990) Stabilizability of integrodifferential parabolic equations, J. Integral Equations Appl. 2, 281-304.

Engel, K.-J., and Nagel, R. (2000) One-Parameter Semigroups for Linear Evolution Equations, Springer-Verlag, New York.

Hadd, S., and Idrissi, A. (2005) Regular linear systems governed by systems with state, input and output delays, IMA J. Math. Control Inform. 22, 423-439.

Hadd, S., Idrissi, A., and Rhandi, A., (2005) The regular linear systems associated to the shift semigroups and application to control delay systems, submitted.

Hale, J.K., and Verduyn Lunel, S.M. (2001) Effects of small delays on stability and control, in H. Bart, I. Gohberg and A. C. M. Ran (Eds.): 'Operator Theory and Analysis (Proc. Amsterdam, 1997),' Oper. Theory Adv. Appl. 122, Birkäuser, Basel, pp. 275-301.

Hinrichsen, D., and Pritchard, A.J. (1994) Robust stability of linear evolution operators on Banach spaces, SIAM J. Control Optim. 32, 1503-1541.

Ichikawa, A. (1982) Quadratic control of evolution equations with delays in control, SIAM J. Control Optim. 20, 645-668.

Jacob, B. (1995) Time-varying infinite dimentional state-space systems, Ph.D. thesis, Bremen.

Jeong, J.M. (1991) Stabilizability of retarded functional-differential equation in Hilbert spaces, Osaka J. Math. 28, 347-365. 
Kowalewski, A. (2003) Time-optimal control of hyperbolic systems with deviating arguments, Internat. J. Control 76, 557-565.

Kowalewski, A., and Krakowiak, A. (2001) Boundary control of retarded parabolic systems with the non-homogeneous Dirichlet boundary conditions, IMA J. Math. Control Inform. 18, 381-393.

Lasiecka, I., and Triggiani, R. (2000) Control Theory for Partial Differential Equations: Continuous and Approximation Theories, Volume I, Cambridge University Press, Cambridge.

Nakagiri, S., and Yamamoto, M. (2001) Feedback stabilization of linear retarded systems in Banach spaces, J. Math. Anal. Appl. 262, 160-178.

Pritchard, A.J., and Salamon, D. (1985) The linear quadratic control problem for retarded systems with delays in control and observation, IMA J. Math. Control Inform. 2, 335-362.

Salamon, D. (1987) Infinite-dimentional linear system with unbounded control and observation: a functional analytic approach, Trans. Amer. Math. Soc. 300 (2), 383-431.

Schnaubelt, R. (2002a) Well-posedness and asymptotic behaviour of non-autonomous linear evolution equations, A. Lorenzi, B. Ruf (Eds.): "Evolution Equations, Semigroups and Functional Analysis," Birkhäuser, pp. 311-338.

Schnaubelt, R. (2002b) Feedbacks for non-autonomous regular linear systems, SIAM J. Control Optim. 41, 1141-1165.

Schnaubelt, R. (2004) Parabolic evolution equations with asymptotically autonomous delay, Trans. Amer. Math. Soc. 356, 3517-3543.

Staffans, O.J. (2004) Well-Posed Linear Systems Part I: General Theory, Cambridge University Press.

Vinter, R.B., and Kwong, R.H. (1981) The infinite time quadratic control problem for linear systems with state and control delays: An evolution equation approach, SIAM J. Control Optim. 19, 139-153.

Weiss, G. (1989a) Admissible observation operators for linear semigoups, Israel J. Math. 65, $17-43$.

Weiss, G. (1989b) Admissibility of unbounded control operators, SIAM J. Control Optim. 27, $527-545$.

Weiss, G. (1994a) Transfer functions of regular linear systems. Part I: Characterization of regularity, Trans. Amer. Math. Soc. 342, 827-854.

Weiss, G. (1994b) Regular linear systems with feedback, Math. Control Signals Systems 7, 23-57. 